\title{
Simultaneous Investigation of the Liquid Transport and Swelling Performance during Tablet Disintegration
}

\author{
Mohammed Al-Sharabi ${ }^{\mathrm{a}}$, Daniel Markl ${ }^{\mathrm{b}, \mathrm{c}}$, Theona Mudley ${ }^{\mathrm{a}}$, Prince Bawuah ${ }^{\mathrm{a}}$, \\ Anssi-Pekka Karttunen ${ }^{\mathrm{d}}$, Cathy Ridgway ${ }^{\mathrm{e}}$, Patrick Gane ${ }^{\mathrm{f}}$, Jarkko Ketolainen ${ }^{\mathrm{d}}$, \\ Kai-Erik Peiponeng, Thomas Rades ${ }^{\mathrm{h}}$, J. Axel Zeitler, ${ }^{\mathrm{a}, *}$ \\ ${ }^{a}$ Department of Chemical Engineering and Biotechnology, University of Cambridge, \\ Cambridge, CB3 OAS, UK \\ ${ }^{b}$ Strathclyde Institute of Pharmacy and Biomedical Sciences, University of Strathclyde, 161 \\ Cathedral Street, G4 ORE Glasgow, UK \\ ${ }^{c}$ EPSRC Centre for Innovative Manufacturing in \\ Continuous Manufacturing and Crystallisation, University of Strathclyde, 99 George Street, \\ G1 1RD Glasgow, UK \\ ${ }^{d}$ School of Pharmacy, Promis Centre, University of Eastern Finland, P.O. Box 1627, \\ FI-70211 Kuopio, Finland \\ ${ }^{e}$ Omya International AG, Forschackerstrasse 6, CH 4622 Egerkingen, Switzerland \\ ${ }^{f}$ Aalto University, School of Chemical Engineering, Department of Bioproducts and \\ Biosystems, FI-00076 Aalto, Finland \\ ${ }^{g}$ Institute of Photonics, University of Eastern Finland, P.O. Box 111, FI-80101 Joensuu, \\ Finland \\ ${ }^{h}$ Department of Pharmacy, University of Copenhagen, Universitetsparken 2, DK-2100 \\ Copenhagen, Denmark
}

\begin{abstract}
Fast disintegrating tablets have commonly been used for fast oral drug delivery to patients with swallowing difficulties. The different characteristics of the pore structure of such formulations influence the liquid transport through the tablet and hence affect the disintegration time and the release of the drug in the body. In this work, terahertz time-domain spectroscopy and terahertz pulsed imaging were used as promising analytical techniques to quantitatively analyse the impact of the structural properties on the liquid uptake and swelling rates upon contact with the dissolution medium. Both the impact of porosity and formulation were investigated for theophylline and paracetamol based tablets. The drug substances were either formulated with functionalised calcium carbonate (FCC) with porosities of $45 \%$ and $60 \%$ or with microcrystalline cellulose (MCC)
\end{abstract}

\footnotetext{
* Corresponding author
} 
with porosities of $10 \%$ and $25 \%$. The terahertz results reveal that the rate of liquid uptake is clearly influenced by the porosity of the tablets with a faster liquid transport observed for tablets with higher porosity, indicating that the samples exhibit structural similarity in respect to pore connectivity and pore size distribution characteristics in respect to permeability. The swelling of the FCC based tablets is fully controlled by the amount of disintegrant, whereas the liquid uptake is driven by the FCC material and the interparticle pores created during compaction. The MCC based formulations are more complex as the MCC significantly contributes to the overall tablet swelling. An increase in swelling with increasing porosity is observed in these tablets, which indicates that such formulations are performance-limited by their ability to take up liquid. Investigating the effect of the microstructure characteristics on the liquid transport and swelling kinetics is of great importance for reaching the next level of understanding of the drug delivery, and, depending on the surface nature of the pore carrier function, in turn controlling the performance of the drug mainly in respect to dissolution in the body.

Keywords: Porous media, pharmaceutical tablets, terahertz, microstructure, liquid transport, swelling, disintegration

\section{Introduction}

Pharmaceutical tablets are the most widely used dosage form for orally administering a drug to a patient (Han et al., 2018). These oral solid dosage forms can be formulated as immediate release tablets for fast release of their drug content (full disintegration within 10 to 60 min of ingestion depending on the regulatory requirements that apply) (Corveleyn \& Remon, 1997; European Medicines Agency, 2008) or modified release tablets for a delayed or controlled liberation of the drug over a specified period of time (Gaikwad et al., 2018). A special case of an immediate-release delivery system is a fast disintegrating tablet (FDT), which is designed to rapidly disintegrate in a small amount of saliva (e.g. 1-2 ml) (Fu et al., 2004). This method can achieve better patient 
compliance and enhanced treatment for people with dysphagia (swallowing difficulty), such as paediatric, geriatric, psychiatric, paralysed and bedridden patients (Bandari et al., 2014; Nishiyama et al., 2016; Gulsun et al., 2018). FDTs, orally dispersible/disintegrating tablets as described by the European Pharmacopeia, should be designed to disintegrate in less than 3 min before swallowing (Chandrasekhar et al., 2009).

The fast disintegration process of FDTs is of great importance as it ensures rapid exposure of the drug to the dissolution medium and hence increases the rate of dissolution, particularly for formulations with poorly soluble drugs, which in turn can enhance the bioavailability and efficacy of drugs (Dukić-Ott et al., 2007; Parkash et al., 2011). Tablet disintegration involves a number of mechanisms including liquid penetration/wicking (Markl \& Zeitler, 2017), swelling (Yassin et al., 2015b) and strain recovery (Quodbach \& Kleinebudde, 2016). The rate of liquid transport through the porous tablet significantly influences the disintegration of the tablet and hence the dissolution rate (Quodbach \& Kleinebudde, 2014). In particular, liquid penetration into rapidly disintegrating porous tablets is the first, and often the rate-determining, step of the disintegration process of such materials (Nogami et al., 1967). The liquid imbibition into the pore space of the tablet does not necessarily cause the breakage of the tablet, but activates other disintegration mechanisms, such as the swelling of the disintegrant particles (Desai et al., 2016). The key characteristics of the pore structure properties of a tablet, such as porosity, connectivity and pore shape, size and orientation, thus control the rate of liquid transport through the pore space of the tablet (Markl et al., 2018b). Tablet disintegration can thus be accelerated by optimising the pore space (e.g. increasing the porosity, pore connectivity and permeability) while maintaining high mechanical stability (Chauhan et al., 2018; Pabari \& Ramtoola, 2012). This can be achieved through modifying the inter-particle pore space by adjusting the compaction procedure (e.g. compaction pressure) and through incorporating highly porous excipients, fillers in particular, in tablet formulations (Markl et al., 2017a). In order to better understand the interactions between formulation, the manufac- 
turing settings and the disintegration performance, it is essential to quantify the disintegration mechanism in more detail.

A significant number of studies have been conducted by researchers using a range of techniques including high-speed video imaging (Desai \& Heng, 2012), gravimetric techniques (Nogami et al., 1966; Peppas \& Colombo, 1989) and magnetic resonance imaging (MRI) (Tritt-Goc \& Kowalczuk, 2002; Quodbach et al., 2014) to investigate the liquid transport into porous pharmaceutical powder compacts and the subsequent disintegration process of such materials upon contact with the dissolution medium. Terahertz pulsed imaging (TPI) coupled with a customised flow cell was previously used successfully to study the fast transport of liquid into porous media, such as powder compacts, and to investigate the impact of the microstructure characteristics on the liquid transport kinetics (Markl \& Zeitler, 2017). Initially, Obradovic et al. (2007) developed a method to study solvent penetration and swelling kinetics in different polymeric materials. Yassin et al. (2015a,b) and Markl et al. (2017c, 2018c) have also used TPI to investigate the liquid ingress and swelling kinetics in a range of pharmaceutical powder compacts.

This work aimed at developing a deeper understanding of the rapid liquid transport kinetics of FDTs for two different model drugs using terahertz methods. The terahertz methods will be used for the first time to investigate the effect of the microstructure characteristics of the tablet on the liquid imbibition and swelling of complex formulations containing either theophylline or paracetamol.

\section{Materials and Methods}

\subsection{Materials}

The pharmaceutical tablets are composed of either functionalised calcium carbonate (FCC) (Omyapharm ${ }^{\circledR}$; Omya International AG, Oftringen, Switzerland) or microcrystalline cellulose (MCC) (Avicel PH-101, FMC BioPolymer, Philadelphia, USA) as a filler (also as a binder in the case of MCC) and 10\% of 
either theophylline anhydrous (Sigma-Aldrich, Steinheim, Germany) or paracetamol (Hangzhou Dayangchem CO. Ltd, Hangzhou, China) as an API. The solubility of theophylline and paracetamol in water at $25^{\circ} \mathrm{C}$ is $7360 \mathrm{mg} \mathrm{l}^{-1}$ and $14000 \mathrm{mg} \mathrm{l}^{-1}$, respectively (Yalkowsky et al., 2010). All the formulations contain $2 \%$ of croscarmellose sodium (CCS) (Vivasol, JRS Pharma, Rosenberg, Germany) as a swelling disintegrant, which facilitates the disintegration process of the tablet upon contact with the dissolution medium. The FCC based tablets have nominal porosities of $45 \%$ and $60 \%$ and the MCC based tablets have nominal porosities of $10 \%$ and $25 \%$ (Table 1). The higher porosity of the FCC based formulation is due to the higher intrinsic porosity of the FCC particles as outlined previously (Markl et al., 2018c).

MCC is one of the most common filler/binder excipients in use, it can be used in the preparation of FDTs due to its ability to absorb water into the interparticle voids as well as due to the diffusion of water into the inter-polymer space of cellulose (Thoorens et al., 2014; Ridgway et al., 2017). However, the maximum porosity that can be achieved for a tablet with sufficient tensile strength is limited for MCC tablets (Markl et al., 2017b). This porosity limitation can have an impact on the rate of liquid uptake and hence the disintegration of the MCC based FDTs. Beside MCC, FCC is another promising excipient for formulating highly porous FDTs (Markl et al., 2018c). Ridgway et al. (2004) developed FCC with high porosities (up to $60 \%$ ) and large surface areas to improve the liquid uptake capacity and increase the liquid absorption rate (10 times higher than the conventional calcium carbonate). FCC can still be considered as a relatively new pharmaceutical excipient, it has shown great potential for the production of the FDTs due to its high mechanical strength, suitability for direct compaction, intra-particle porosity and short tablet disintegration time (Stirnimann et al., 2013).

\subsection{Tablet Compaction}

A compaction simulator (PuuMan PCS-1; PuuMan Ltd, Kuopio, Finland) was used to compact the powder of each formulation into flat-faced round tablets 
with a diameter of $10 \mathrm{~mm}$ and a thickness of $1.6 \mathrm{~mm}$. The different targeted porosities of the tablets were achieved by changing the weight of the formulation powder and maintaining fixed tablet diameter and thickness. The true density of the formulation, $\rho_{\text {true }}$, was calculated using literature values of the true densities of the constituents and their ratios in each formulation (Mesnier et al., 2013; Bawuah et al., 2016; Markl et al., 2017b). The mass, $m$, diameter, D, and thickness, $H$, of the compacted tablet were measured to calculate the tablet apparent density, $\rho_{\text {apparent }}$. The nominal porosity, $f_{\text {nominal }}$, was then calculated using the relative density of the tablet, $\rho_{\text {relative }}$, which is the ratio of $\rho_{\text {apparent }}$ to $\rho_{\text {true }}$ of the tablet as defined in Equation 1 (Markl et al., 2017b).

$$
\begin{aligned}
f_{\text {nominal }}(\%) & =\left(1-\rho_{\text {relative }}\right) \times 100=\left(1-\frac{\rho_{\text {apparent }}}{\rho_{\text {true }}}\right) \times 100 \\
& =\left(1-\frac{4 m}{D^{2} H \rho_{\text {true }} \pi}\right) \times 100
\end{aligned}
$$

Table 1: The different formulations of the tablets used for the terahertz measurements.

\begin{tabular}{|c|c|c|c|}
\hline Formulation & API & $f_{\text {nominal }}(\%)$ & Batch code \\
\hline \multirow{3}{*}{ FCC } & \multirow{3}{*}{ Theophylline } & 45 & FT45 \\
\cline { 3 - 4 } & & 60 & FT60 \\
\cline { 3 - 4 } & \multirow{2}{*}{ Paracetamol } & 45 & FP45 \\
\cline { 3 - 4 } & & 60 & FP60 \\
\hline \multirow{3}{*}{ MCC } & \multirow{2}{*}{ Theophylline } & 10 & MT10 \\
\cline { 3 - 4 } & \multirow{2}{*}{ Paracetamol } & 25 & MT25 \\
\cline { 3 - 4 } & & 10 & MP10 \\
\cline { 3 - 4 } & & 25 & MP25 \\
\hline
\end{tabular}

\subsection{Terahertz Time-Domain Spectroscopy and Imaging}

A commercial terahertz system (TeraPulse 4000, Teraview Ltd., Cambridge, UK) was used to conduct all the terahertz measurements in either transmission or reflection mode. Terahertz time-domain spectroscopy (THz-TDS) measurements in transmission mode refer to the terahertz-based porosity measurements 


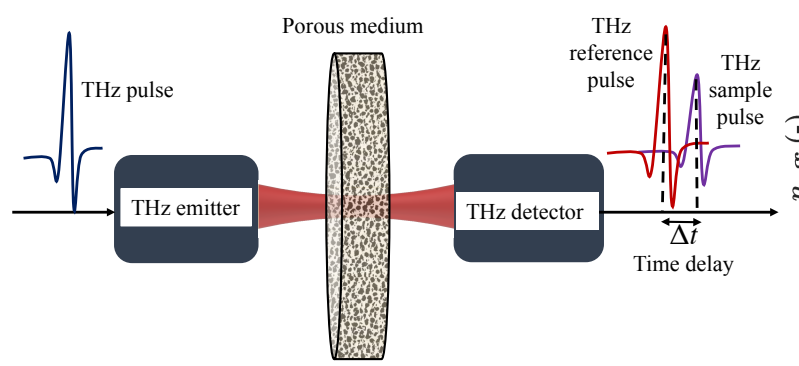
swelling measurements.

\subsubsection{Terahertz-based Porosity Measurements} index, $n_{\text {eff }}$, is determined (Chakraborty et al., 2017). while TPI measurements in reflection mode refer to the liquid transport and

As outlined above in section 2.3, the terahertz waveforms of the sample and the reference, i.e. no sample is present in the transmission measurement chamber, were acquired in the time-domain (TD) using the terahertz spectrometer in transmission mode (Figure 1). The THz-TDS measurements were conducted for 20 tablets of each batch. 20 waveforms were co-averaged for each single measurement of the sample and the reference to improve the signal-to-noise ratio. The duration of each measurement was $40 \mathrm{~s}$. The transmission system was purged with dry nitrogen gas throughout the measurements to minimise the impact of the water vapour on the transmission measurements (Bawuah et al., 2018). Any sample such as the porous medium of the tablet causes a delay of the terahertz pulse in the time-domain, $\Delta t$, (see Figure 1). The delay is defined from the temporal difference between a reference of the pulse propagating through the empty spectrometer, and the sample pulse. Based on $\Delta t$ the effective refractive

Figure 1: The terahertz transmission measurement configuration for studying the tablet pore structure.

The time-domain waveforms of the sample and reference pulses can be con- verted to the frequency-domain (FD) by applying a fast Fourier transform (FFT)

to calculate the frequency-dependent effective refractive index, $n_{\mathrm{eff}}(\nu)$ (Markl 
et al., 2017b). Effective medium approximation (EMA) models, such as the Bruggeman model, can then be used to predict the porosity of the tablet using the measured values of $n_{\mathrm{eff}}(\nu)$ (Bawuah et al., 2014b). It has been shown that the anistropic Bruggeman model (AB-EMA) is suitable for predicting the porosity of porous media with non-spherical pore shapes due to the utilisation of a depolarisation factor, $L$ (Markl et al., 2017b). Note that an $L$ value of $1 / 3$ yields the traditional Bruggeman model (TB-EMA), which assumes spherical shapes of pores (Bawuah et al., 2014a). A structural parameter, the so-called $S_{\text {a }}$ parameter, can also be obtained from the THz-TDS measurements. This parameter reflects the anisotropy in the microstructure of pharmaceutical tablets in terms of the arrangement of the pores relative to the direction of the propagation of the terahertz radiation (Markl et al., 2018a). In this study, the terahertz pulse always propagated through the tablet in axial direction (same direction as the compaction direction).

\subsubsection{Terahertz Pulsed Imaging}

The TPI measurements were performed to investigate the one-dimensional (1D) liquid transport and swelling kinetics in fast disintegrating tablets to better understand their disintegration process upon exposure to the dissolution medium. A customised flow cell with the commercial THz-TDS system was used to acquire the liquid transport measurements in reflection configuration. The flow cell was first designed and utilised by Yassin et al. (2015a) to study the water imbibition and swelling of various MCC based tablets to evaluate the disintegration performance of such tablets. Markl et al. (2018c) modified the flow cell to improve the data acquisition and the accuracy of the measurements of the rapid water absorption into highly porous FCC tablet compacts. For all measurements 6 tablets of each batch, except for the batches of FP45 (4 tablets), FT60 (5 tablets) and MT10 (5 tablets), were used for the TPI measurements. Measurement principle. The TPI method exploits the difference between the refractive indices of the dry material and liquid, which results in a reflection of the terahertz pulse from the interface between the two media. By tracking 
this reflection as a function of time it is possible to quantify the wicking process within the porous tablet (Yassin et al., 2015b). Differences in the refractive index can be due to i) a density difference within the same medium, or ii) a change in the type of the medium through which the pulse of terahertz radiation propagates (Zeitler, 2016). Therefore, it is possible to study the liquid transport into pharmaceutical tablets due to the difference in the refractive indices of the dry tablet and material wetted with the dissolution medium. The tracking of the peaks from the water penetration front and swelling was performed using customised MATLAB (MathWorks, Massachusetts, USA) code. The rate of liquid penetration through the tablet was determined by tracking the movement of the reflection peak of the water front. The rate of swelling was captured by tracking the shift in the positive peak of the reflection from the tablet back face relative to its original position. This shift is due to an increase in the tablet thickness upon swelling. The reflections from the different interfaces at the front and back faces of the tablet as well as the swelling and liquid penetration front are illustrated in Figure 2. 

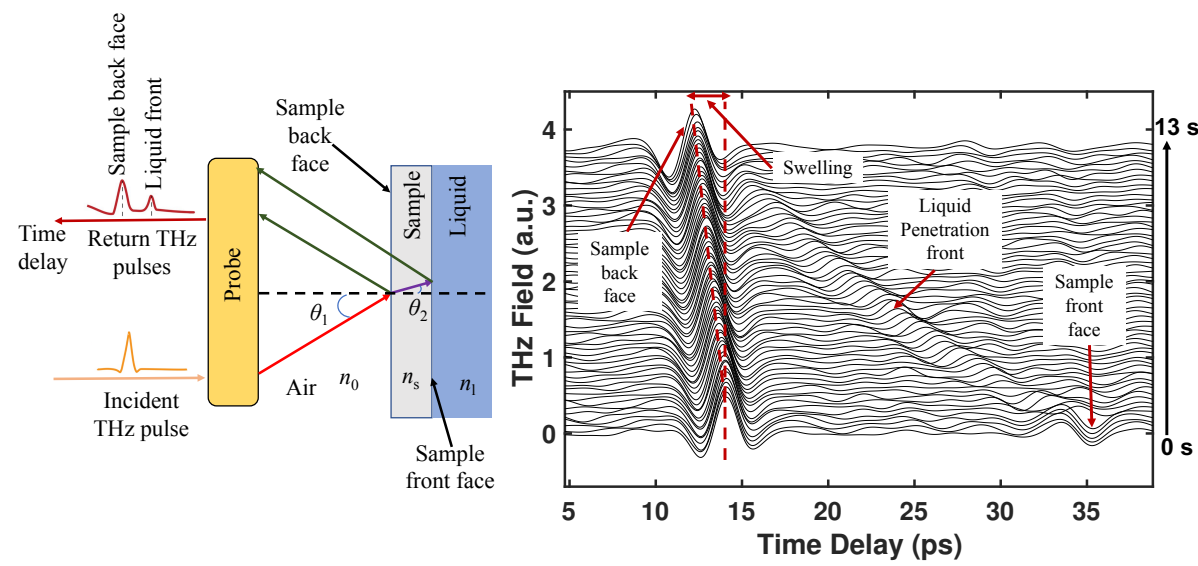

Figure 2: The measurement principle of TPI in a reflection setup. The waterfall plot shows the terahertz electric field of the deconvolved terahertz waveforms as a function of the time delay for a tablet from batch FT45. Every third waveform was plotted with an offset of 0.02 a.u. between each waveform. The first waveform from the bottom of the waterfall plot corresponds to the first TPI measurement whereas the last measurement at the top is the last measurement upon the full hydration of the tablet. The total duration of the complete hydration and disintegration of the tablet upon contact with the dissolution medium is indicated in seconds on the right of the waterfall plot. $n_{0}, n_{\mathrm{s}}$ and $n_{1}$ are the refractive indices of air, sample and liquid, respectively. $\theta_{1}$ and $\theta_{2}$ are the angles of incidence and refraction, respectively.

Sample preparation and flow cell setup. Each tablet was placed in a cylindrical polyether ether ketone (PEEK) sample holder, which was then placed in the flow cell (Figure 3). A round piece of Blu Tack ${ }^{\circledR}$ was then placed on the top of the sample with two O-rings placed on the Blu Tack ${ }^{\circledR}$ to keep the sample stationary and provide the required sealing during the measurement. Another piece of Blu Tack ${ }^{\circledR}$ was used to seal between the sample holder and the flow cell. A window made of polyethylene (PE) was used to close and seal the flow cell as PE is transparent to terahertz radiation and therefore the signal loss is minimised. The flow cell was placed on a mounting stage to accurately position the flow with respect to the terahertz reflection probe. The flow cell was connected to a peristaltic pump (530SN, Watson-Marlow Ltd, Falmouth, UK), which has a low-pulse pump head for a smooth water flow to the cell with minimised pressure fluctuations (Markl et al., 2018c). The flow cell is equipped 
with mini ball valves to adjust the flow into and out of the flow cell.

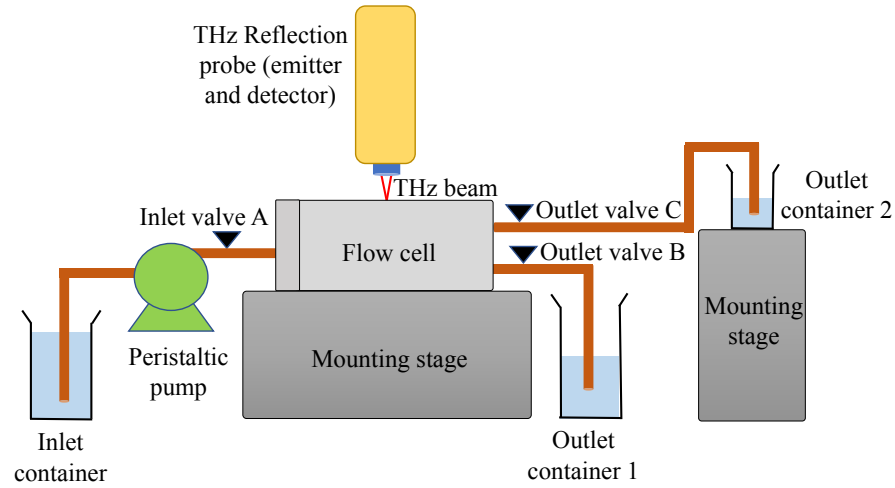

Figure 3: The flow cell setup used for monitoring the liquid ingress through the tablet using TPI.

Experimental procedure and data acquisition. The terahertz signals were acquired at a waveform rate of $15 \mathrm{~Hz}$ using a reflection probe with a focal length of $18 \mathrm{~mm}$. First, baseline waveforms, i.e. measurement of air, and a reference measurement of a mirror were acquired. 500 waveforms were co-averaged for determining the baseline and reference measurements. This was carried out prior to every measurement of a tablet.

The experiment started by opening valves $\mathrm{A}$ and $\mathrm{B}$ and closing valve $\mathrm{C}$ to fill the cell with water. Water was pumped to the flow cell at rate of 10 revolutions

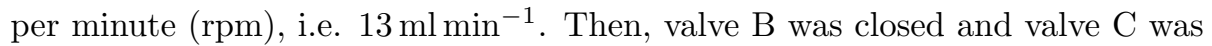
opened to remove air bubbles trapped in the cell and start the hydration of the tablet. The sample measurements were acquired just before opening valve $\mathrm{C}$. The pump was turned off and the sample measurements were stopped once the tablet has fully disintegrated, which is indicated by the disappearance of the positive peak corresponding to the reflection of the terahertz signal at the back face of the tablet.

Data processing. The raw terahertz waveforms acquired from the TPI measurements of each tablet were first deconvolved using MATLAB. This processing included removal of noise, and contrast enhancement to facilitate the analysis 
of the raw TPI data. Deconvolving the measured reflection waveforms is an essential step in the processing of the raw TPI data for highlighting the reflection peaks from the different interfaces, particularly the water front peak. The deconvolution is a simple division of the raw sample signal, $\operatorname{FFT}[s(n)]$, by the reference signal, $\mathrm{FFT}[r(n)]$, in the FD and using a double Gaussian band-pass filter, $\mathrm{FFT}\left[f_{\mathrm{DG}}\right]$, to reduce the amplification of noise as given in Equations 2 (Zeitler \& Shen, 2012) and 3 (Markl et al., 2018c). The FFT[·] operator indicates a fast Fourier transform. The deconvolved signal in the FD was then converted into the TD using an inverse Fourier transform.

$$
\begin{gathered}
\text { Deconvolved signal }=\mathrm{FFT}^{-1}\left[\frac{\mathrm{FFT}[s(n)]}{\mathrm{FFT}[r(n)]} \times \mathrm{FFT}\left(f_{\mathrm{DG}}\right)\right] \\
f_{\mathrm{DG}}(t)=\frac{1}{\mathrm{HF}} \exp \left(-\frac{t^{2}}{\mathrm{HF}^{2}}\right)-\frac{1}{\mathrm{LF}} \exp \left(-\frac{t^{2}}{\mathrm{LF}^{2}}\right)
\end{gathered}
$$

where $t$ is the optical time delay, and HF and LF are the high and low frequency bounds of the double Gaussian band-pass filter, respectively, which define the pulse width

\subsection{X-ray Microcomputed Tomography}

X-ray microcomputed tomography $(\mathrm{X} \mu \mathrm{CT})$ measurements were conducted using a Skyscan 1172 scanner (Bruker, Antwerp, Belgium) to investigate the presence of cracks and/or agglomerates in the tablet. A tablet was fixed on the sample holder using a double-sided sticky tape and then placed in the $\mathrm{X} \mu \mathrm{CT}$ instrument. Shadow projection images were collected with a resolution of $3.98 \mu \mathrm{m}$ at an angular step, i.e. rotation increment, of $0.25^{\circ}$ over $180^{\circ}$ using an aluminium filter (Al $0.5 \mathrm{~mm})$. 10 frames were averaged per position with an exposure time of $4.5 \mathrm{~s}$. The projected images were then reconstructed using NRecon software (Bruker, Version: 1.7.4.2) to obtain cross-sections of the sample. DataViewer software (Bruker, Version: 1.5.3.4) was then utilised to visualise the cross-sectional images of the sample. 


\subsection{Mercury Porosimetry}

Mercury intrusion measurements were made using an Autopore V mercury porosimeter (Micromeritics Instrument Corporation, Norcross, GA, U.S.A.). One tablet of each batch was used per measurement. The maximum applied pressure of mercury was $414 \mathrm{MPa}$, equivalent to a Laplace throat diameter of $4 \mathrm{~nm}$. The equilibration time at each of the increasing applied pressures of mercury is set to $20 \mathrm{~s}$. Parameterising the elastic behaviour in the data can be made as part of the overall data correction during the mercury intrusion comprising the more commonly known effects of compression of mercury and expansion of the penetrometer (Gane et al., 1996). This is performed conveniently using the software Pore-Comp (a software program developed by and obtainable from the Environmental and Fluids Modelling Group, University of Plymouth, U.K.), in which the following equation is applied:

$$
\begin{aligned}
V_{\text {int }}= & V_{\text {obs }}-\delta V_{\text {blank }}+\left[0.175\left(V_{\text {bulk }}^{1}\right) \log _{10}\left(1+\frac{P}{1820}\right)\right] \\
& -V_{\text {bulk }}^{1}\left(1-\Phi^{1}\right)\left(1-\exp \left[\frac{\left(P^{1}-P\right)}{M_{\mathrm{ss}}}\right]\right)
\end{aligned}
$$

where $V_{\text {int }}$ is the volume of intrusion into the sample, $V_{\text {obs }}$ the intruded mercury volume reading, $\delta V_{\text {blank }}$ the change in the blank run volume reading, $V_{\text {bulk }}^{1}$ the sample bulk volume at atmospheric pressure, $P$ the applied pressure, $\Phi^{1}$ the porosity at atmospheric pressure, $P^{1}$ the atmospheric pressure and $M_{\mathrm{ss}}$ the bulk modulus of the solid sample (Gane et al., 1996).

\section{Results and Discussion}

The pore structure properties, such as porosity as well as $S_{\text {a }}$ parameter, will be presented first. Then, the liquid penetration and swelling profiles will be discussed to understand the impact of porosity as well as drug solubility on the liquid transport kinetics. Finally, a link between the structural properties and the parameters of the liquid transport kinetics will be demonstrated. 


\subsection{Analysis of Pore Structure}

The frequency dependent $n_{\text {eff values obtained from the THz-TDS measure- }}$ ments were used for the calculation of the THz-TDS porosity of the tablets using the AB-EMA model as well as the calculation of the $S_{\text {a }}$ parameter. For subsequent analysis the $n_{\text {eff }}$ was selected at a frequency of $1.2 \mathrm{THz}$ (Figure 1) for all formulations. This frequency was chosen given that no significant dispersion in $n_{\text {eff }}$ was observed, i.e. $n_{\text {eff }}$ is constant for each set of tablets, and there are no characteristic absorption peaks visible. The predicted terahertz porosity using AB-EMA was plotted against the nominal porosity for the different formulations to compare the suitability of the AB-EMA model in predicting the porosity (Figure 4). The depolarisation factor $L$ in the AB-EMA model was determined to be 0.239 for FCC-theophylline tablets, 0.235 for FCC-paracetamol tablets, 0.364 for MCC-theophylline tablets and 0.405 for MCC-paracetamol tablets. AB-EMA performs well and yields an accurate and reliable prediction of porosity due to the accurate selection of the depolarisation factor, particularly for the FCC based tablets where the pores in such tablets are predicted have a non-spherical shape with a perpendicular alignment to the direction of compaction (Markl et al., 2017b). The porosity predicted by AB-EMA will be used as the terahertz porosity for the investigation of the effect of porosity on the liquid transport kinetics.
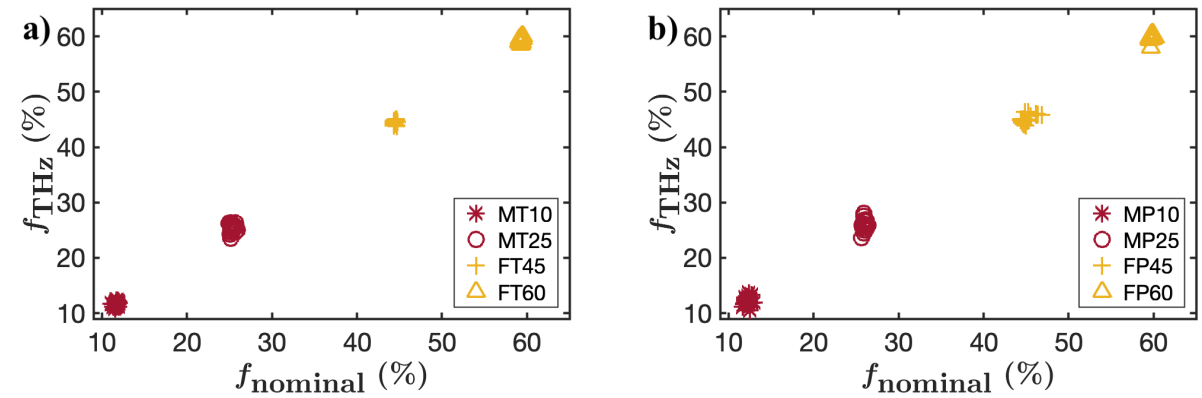

Figure 4: The THz-TDS porosity, $f_{\mathrm{THz}}$, predicted by AB-EMA as a function of the nominal porosity, $f_{\text {nominal }}$, for the formulations containing (a) theophylline and (b) paracetamol.

The values of the $S_{\text {a }}$ parameter (Figure 5 ) suggest that the pores as well as 
the lumped solid constituents are randomly aligned in the majority of cases. A larger variation in the $S_{\text {a }}$ parameter is observed within each batch of the MCC based tablets in comparison to the FCC based tablets. This variation in the $S_{\mathrm{a}}$ parameter might indicate that tablets of the same formulation and porosity can exhibit different arrangements and shapes of pores, and can be attributed to inhomogeneity in the formulation during the mixing process. Further research is required to fully explore the complex interplay between the mixing process, the particle properties, the resulting porosity of the powder compact as well as the physical interpretation of the $S_{\text {a }}$ parameter.
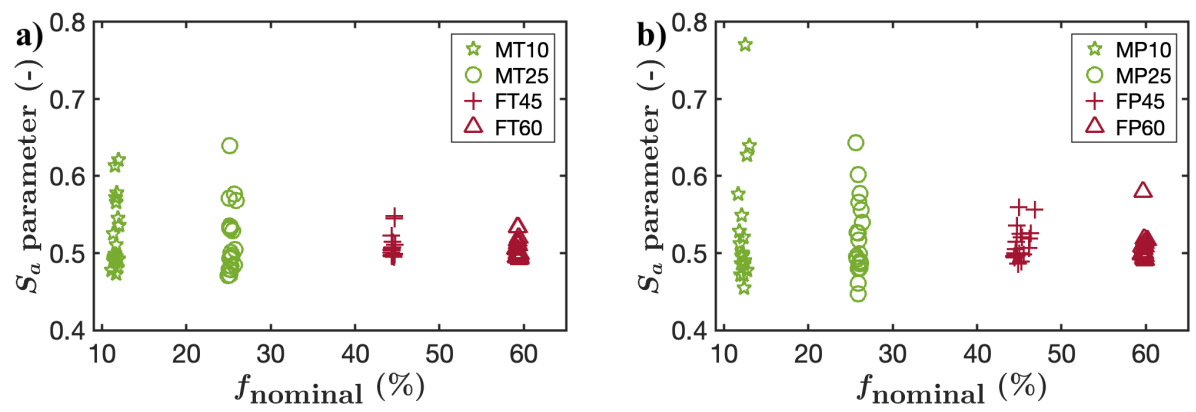

Figure 5: The $S_{\text {a }}$ parameter as a function of the nominal porosity, $f_{\text {nominal }}$, for the formulations containing (a) theophylline and (b) paracetamol.

\subsection{In-situ Monitoring of Liquid Imbibition and Swelling}

The terahertz electric field of the deconvolved terahertz waveforms for the total liquid ingress process of each tablet is displayed as a function of the time delay, i.e. waterfall plot, to visualise the movement of the water penetration front as well as the extent of swelling in the tablet. The waterfall plots of a tablet from each batch are shown in Figures 6 and 7 for the theophylline and paracetamol containing tablets, respectively. The waterfall plots indicate that the total duration for the full hydration and disintegration is less for the higher porosity tablets of the same formulation. 

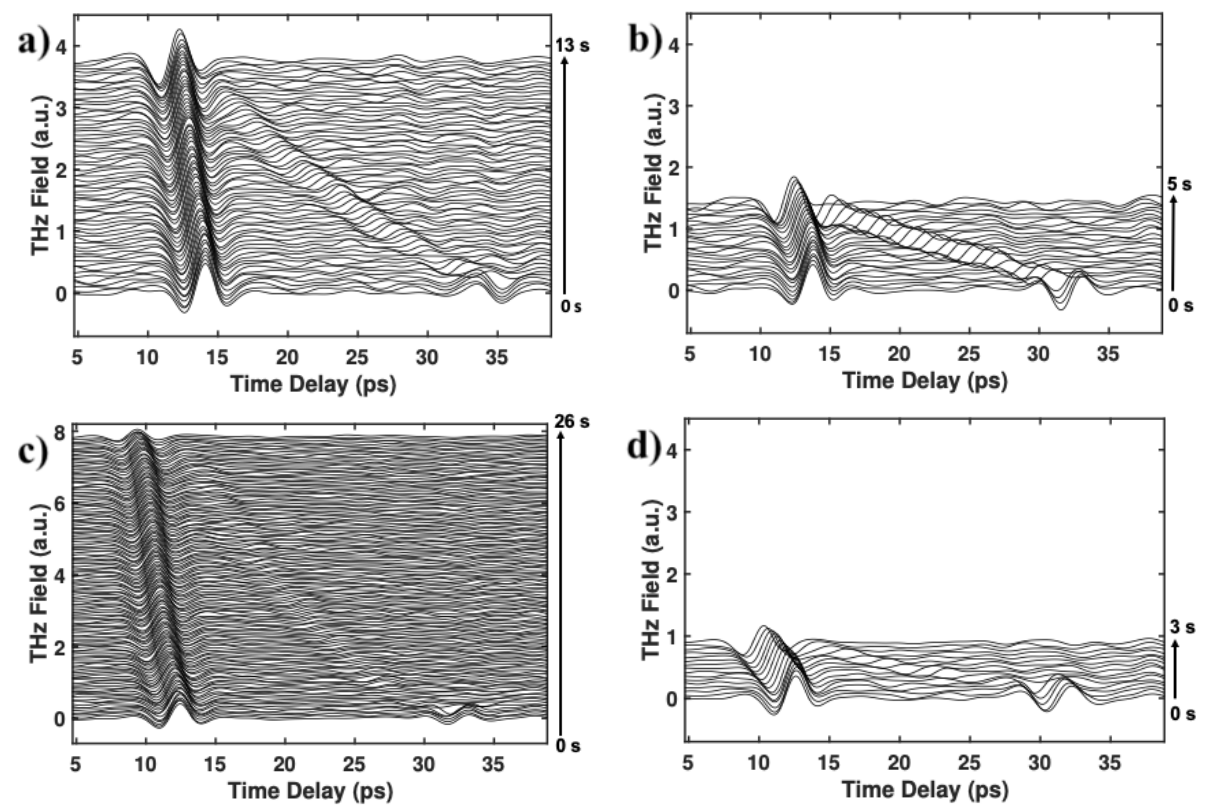

Figure 6: Waterfall plots showing the deconvolved terahertz waveforms of one tablet per batch: (a) FT45, (b) FT60, (c) MT10 and (d) MT25. Every third waveform was plotted with an offset of 0.02 a.u. between each waveform. The total duration of the complete hydration and disintegration of the tablet upon contact with the dissolution medium is indicated in seconds on the right of each waterfall plot. 

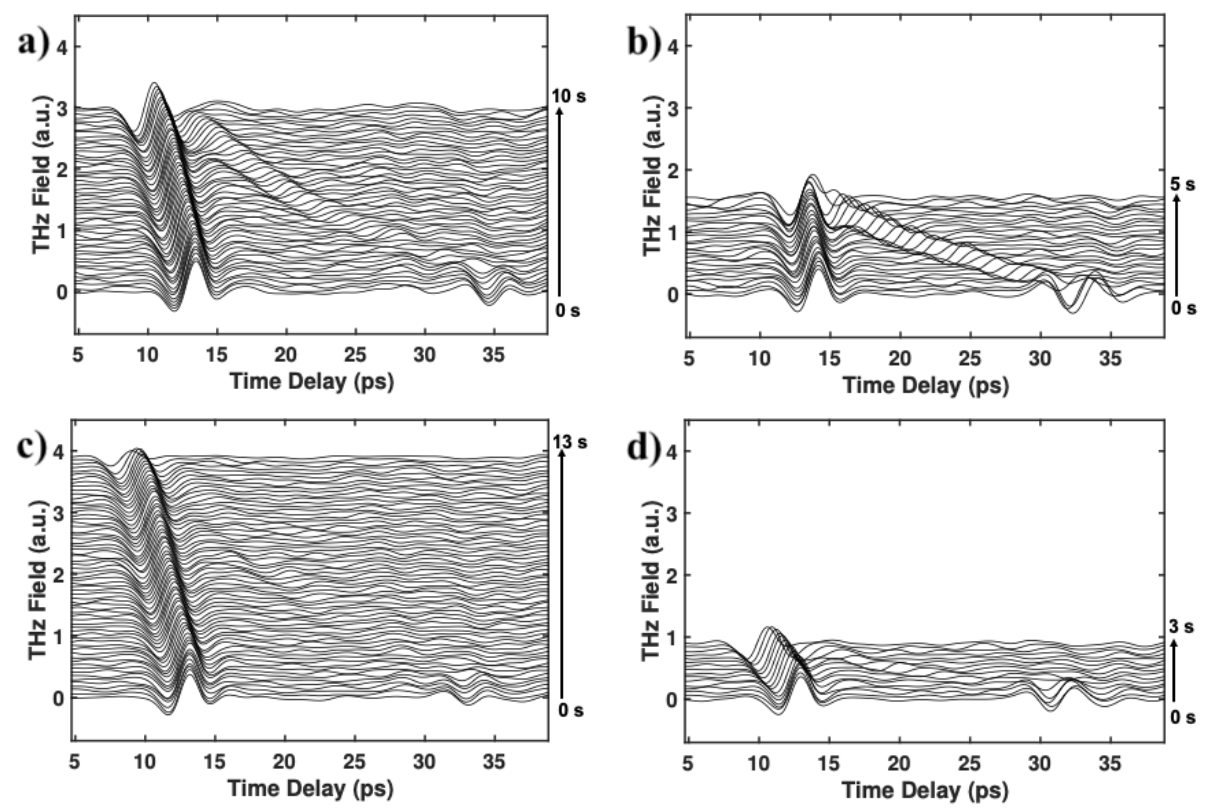

Figure 7: The waterfall plots showing the deconvolved terahertz waveforms of one tablet per each of batch of (a) FP45, (b) FP60, (c) MP10 and (d) MP25. Each third waveform was plotted with an offset of 0.02 a.u. between each waveform. The total duration of the complete hydration and disintegration of the tablet upon contact with the dissolution medium is indicated in seconds on the right of each waterfall plot.

The amplitude of each reflection peak is directly proportional to the reflection coefficient, $r_{12}$, according to the Verdet convention, which is expressed as (Markl et al., 2018c)

$$
r_{12}=\frac{n_{2}-n_{1}}{n_{1}+n_{2}}
$$

In Equation 5 we assume that the terahertz pulse propagates from medium 1 to medium 2 with different refractive indices of $n_{1}$ and $n_{2}$, respectively. We can use this relationship to qualitatively analyse the reflections from the various interfaces occurring in the terahertz waveforms. In terms of the first peak, the terahertz pulse propagates from a medium with a lower refractive index (i.e. air with $\left.n_{1}=1\right)$ to a medium with a higher refractive index $\left(n_{2}=n_{\text {eff }}>1\right.$ of the dry tablet), which results in a positive reflection peak according to Equation 5. Initially, the peak of the reflection from the tablet front face is negative as the 
terahertz beam travels from the dry tablet $\left(n_{1}=n_{\text {eff }}>1\right)$ to air $\left(n_{2}=1\right)$. The wetting of the tablet causes a change of the refractive index resulting in a positive peak of the water front due to the higher refractive index of water $\left(n_{2}=n_{\text {wet }}\right.$ with $n_{\text {eff }}<n_{\text {wet }}<n_{\text {water }} \approx 2.1$ at $1.2 \mathrm{THz}$ ) (Pickwell \& Wallace, 2006) compared to that of the dry tablets used in this study. In general, the absolute difference between the refractive indices of the dry and wetted tablet is directly proportional to the magnitude of the reflection peak from the water front. Two cases could cause a reduction in the magnitude of the reflection peak from the water front: i) a small absolute difference between the refractive indices of the dry tablet and the dissolution medium, and ii) the formation of a water gradient in the tablet during liquid transport through the porous medium.

The waterfall plots show that the magnitude of the amplitude of the water penetration front increases with an increase in the porosity, $f$, due to an increase in the relative difference in the refractive indices of the wetted and dry tablet, i.e. $\left|n_{\text {wet }}-n_{\text {eff }}\right| \propto f$. The batches of the different formulations, excluding the MCC based tablets with $10 \%$ porosity, have a clear and identifiable water penetration front.

According to Equation 5, the MCC based tablets should have a higher reflection amplitude due to the higher relative difference between the $n_{\text {eff }}$ of the MCC based tablets and water when compared to the FCC based tablets. However, this is not the case and the small amplitude of the liquid front reflection in the MCC based tablets with $10 \%$ porosity cannot be attributed to the similarity in the refractive indices of the liquid $\left(n_{\text {water }} \approx 2.1\right.$ at $\left.1.2 \mathrm{THz}\right)$ and sample (MCC based formulation, $n_{\mathrm{eff}} \approx 1.76$ ) as it was possible to resolve it for a smaller relative difference of refractive indices for the FCC tablets with $45 \%$ porosity $\left(n_{\mathrm{eff}} \approx 2\right)$. A previous study (Markl et al., 2017b) concluded that the intraparticle pore structure of FCC does not change during compaction for the very fine pores below $0.014 \mu \mathrm{m}$. These tablets therefore have a very large consistent intra-particle pore space, which is not affected by the compaction. This pore space might be the predominant driver in the liquid imbibition process rather than the inter-particle pore space (Ridgway et al., 2004). If this is the case, 
then a bulk water gradient in FCC based tablets should not be observed. On the contrary, the liquid imbibition process in MCC based formulations is driven by the inter-particle pore space. The inter-particle pore space is not uniform within a tablet as there is a density gradient across the tablet (Markl \& Zeitler, 2017; Djemai \& Sinka, 2006). We therefore speculate that the weak reflection amplitude of the water front can be attributed to the existence of microchannels which promote capillary action that results in the wetting of the dry material before the bulk of the liquid has reached it. As a result a water gradient forms ahead of the imbibition front in the sample and hence the water front is not a clear interface leading to a very weak contrast. In summary, the formation of a water gradient decreases the amplitude of the reflected terahertz pulse in the MCC based tablets with $10 \%$ porosity.

\subsection{Quantification of Liquid Penetration and Swelling Rates}

Figure 8 illustrates the average of the liquid penetration rate for each formulation. The sudden jumps in the liquid penetration profiles of some of the batches are attributed to difficulties in reliably tracking the water front peak during subsequent signal processing. These jumps cause variation in the average liquid penetration profiles of tablets of the same batch, which is represented by the relatively large standard deviation of the liquid penetration profile (e.g. FP45). The porosity of the tablet has a strong impact on the liquid penetration rate. Here we observe that the rate of liquid penetration is higher for the tablets of higher porosities as the larger pore space accelerates the water ingress and the swelling of the MCC particles is not sufficiently fast to constrict the pore mass transport. The API has a significant effect on the liquid penetration kinetics for the MCC based tablets of $10 \%$ porosity. A slower rate of liquid penetration is observed for the MCC tablets that contain theophylline, which may be explained by the poor solubility of theophylline compared to paracetamol. It is interesting to observe that this effect only becomes evident on the liquid transport in the tablets with low porosity. 

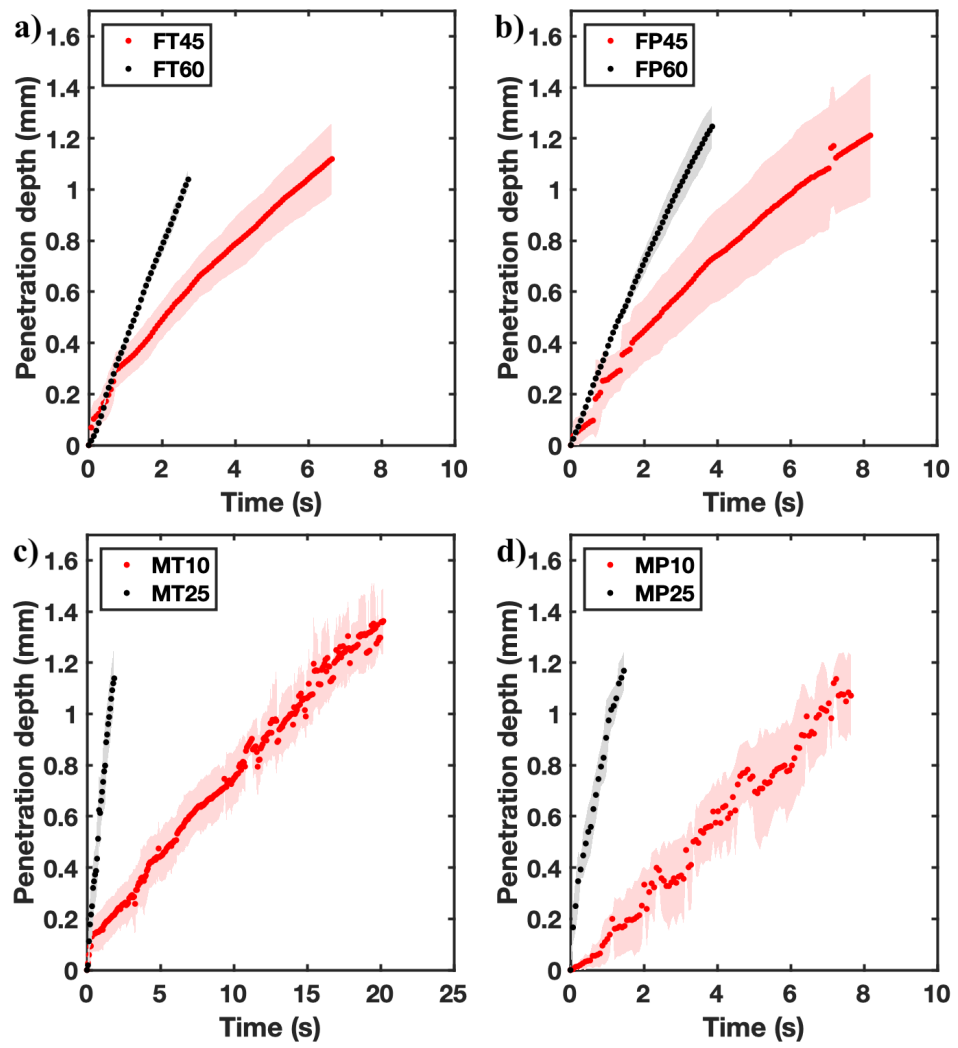

Figure 8: The average liquid penetration profiles of the FCC based tablets ( $\mathrm{a}$ and $\mathrm{b}$ ) and the MCC based tablets (c and d). The shaded area represents the standard deviation for each set. The averaging of the liquid penetration rate of each set was conducted over the shortest time of a complete hydration of the tablet, i.e. time for the fastest tablet to disintegrate.

A large variation in the liquid penetration profiles is observed for the FP45 tablets as indicated by the large standard deviation compared to that of the other batches. The TPI measurements revealed an additional reflection peak that originates from the core of the tablet matrix in a number of tablets from this particular batch. Therefore, $\mathrm{X} \mu \mathrm{CT}$ measurements were conducted on two tablets with and without a defect, i.e. internal cracks and/or agglomerates, to further investigate their microstructure and to determine the source of the additional reflection peak. The $\mathrm{X} \mu \mathrm{CT}$ images (Figure 9) reveal that some of the FP45 tablets contain internal cracks that propagate along agglomerates of 
paracetamol particles. However, the formulations containing theophylline with the same filler and porosity (FCC 45\%) appear homogenous and do not contain internal cracks or agglomerates. We can therefore attribute the additional peaks in the terahertz waveform to the air gaps due to the crack. The tableting behaviour that was observed in our experiments matches previous reports of the crack formation in paracetamol based formulations compared to theophylline formulations (Paul \& Sun, 2017; Chang \& Sun, 2017).
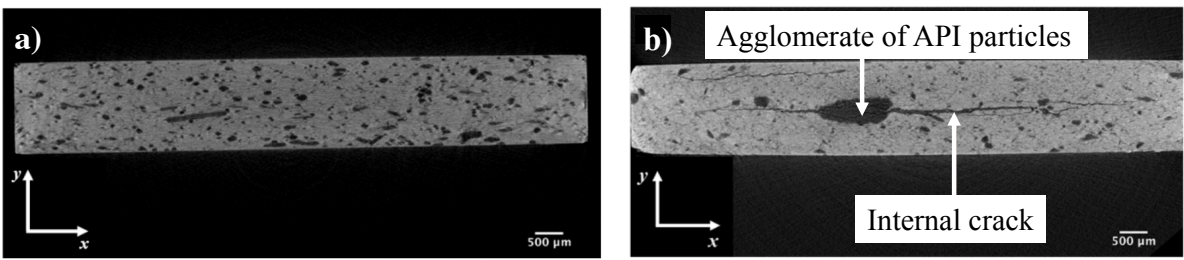

Figure 9: The reconstructed $\mathrm{X} \mu \mathrm{CT}$ images of cross sections of (a) FT45 and (b) FP45. The image in (a) shows a homogenous distribution of API particles, represented by the dark regions, in FT45.

Mercury porosimetry measurements were performed on the MCC and FCC based tablets to further investigate their pore structure. Figure 10 shows the pore size distribution curves based on the data acquired from the mercury porosimetry measurements for the FCC and MCC containing tablets. The peaks of the interparticle pore volume are located at 0.05 and $0.06 \mu \mathrm{m}$ for FT45 and FP45, respectively. For the sets of FT60 and FP60 the peaks of the interparticle pore volume are located at 0.16 and $0.18 \mu \mathrm{m}$, respectively. The peaks of the intraparticle pore volume of the FCC based tablets are very similar for the tablets containing either theophylline or paracetamol with the same porosity. These intraparticle pore volume peaks of the FCC containing tablets lie at 0.02 and $0.03 \mu \mathrm{m}$ for the $45 \%$ and $60 \%$ porosity, respectively. The very fine pores are similar for the two samples of FCC with the $45 \%$ and $60 \%$ porosity below $0.014 \mu \mathrm{m}$ and remain constant under compression, as previously seen (Markl et al., 2017b). However, the pore size region above this diameter displays changes on compaction due to a combination of the effect of particles becoming 
packed tighter together, thus reducing interparticle pore size, and deformation of the surface of FCC at particle-particle contact under pressure, such that these finer contact pores contribute to the intrusion volume across a similar pore size range to that of the larger intraparticle pores.

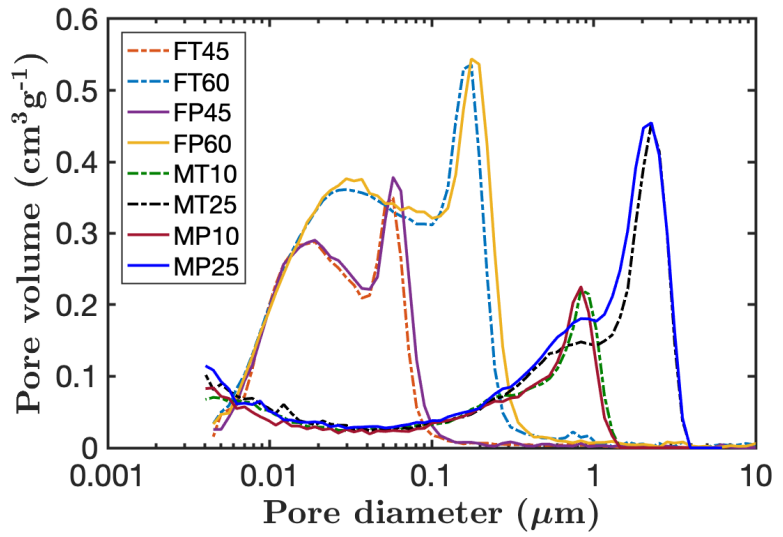

Figure 10: The pore size distributions of both the FCC and MCC based tablets.

The peaks of the interparticle pore volume for the MCC based tablets with $25 \%$ porosity are at $2.27 \mu \mathrm{m}$ for both APIs. For the MCC based tablets with $10 \%$ porosity the interparticle pore volume peaks are observed at $0.84 \mu \mathrm{m}$ for both APIs. The paracetamol containing tablet exhibits the peak towards the finer pore diameters when compared with that of the theophylline containing tablet. The intraparticle pore volume of MP25 is larger than that of MT25 with both peaks located at $0.84 \mu \mathrm{m}$. The paracetamol tablet has thus more fine pores in comparison to the theophylline tablet. There is not a clearly identifiable intraparticle pore volume peak for the MCC based tablets with $10 \%$ porosity.

The optical microscope images of the FCC based tablets post mercury porosimetry measurements (Figure 11) show the presence of an agglomerate of paracetamol particles in the tablet. The inhomogeneity and internal cracks of the FCC based tablets with paracetamol as API can be attributed to the poor flow and compaction properties of paracetamol (Kaerger et al., 2004). The internal cracks were observed for the FCC + paracetamol tablets, and that is why these 
were investigated microscopically. The optical microscope images of the FCC tablets post mercury porosimetry measurements (Figure 11) confirm the presence of agglomerates of paracetamol particles in the tablet. This observation for $\mathrm{FCC}+$ paracetamol does not preclude agglomerates of paracetamol when formulated with MCC excipient (Paul \& Sun, 2017), but the example here of using microscopy after porosimetry is used to illustrate more clearly the grain boundaries between excipient and API, which in the case of cracking, as for the FCC tablet formulation, can lead to macroscale crack-releated inhomogeneities. The presence of such internal defects, agglomerates and/or cracks, influences the liquid penetration and swelling kinetics, which might be the cause of large variations in the liquid penetration profiles of the FP45 tablets as observed in Figure 8.
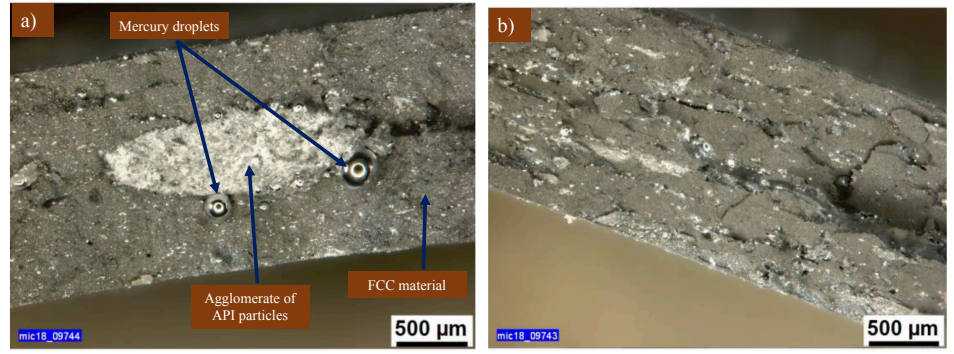

Figure 11: Optical microscope images of FCC based tablets after mercury porosimetry measurements: (a) showing a large agglomerate of API, and (b) the general distribution of smaller agglomerates of API.

Figure 12 shows the average swelling profile for each formulation. Since FCC does not swell (Markl et al., 2018c), the swelling in the FCC based tablets is only due to the presence of the disintegrant, i.e. CCS. A small quantity of CCS ( $2 \%$ by weight in this study) is used in the tablet formulation to promote the rapid breakage of the tablet matrix into smaller particles (Yassin et al., 2015a; Ekmekciyan et al., 2018). All FCC based tablets therefore follow similar swelling kinetics as the concentration of the disintegrant was kept constant. Minor variations in the swelling profiles are due to differences in porosity. The 
extent of swelling of the MCC based tablets is larger than that of the FCC based tablets as the MCC also swells, albeit at different rate compared to CCS, and therefore contributes to the overall swelling of these tablets (Yassin et al., 2015a). The API does not seem to have a significant impact on the extent of swelling upon hydration, which can be attributed to the low concentration of the API in the formulation (10\%). However, there is a variation in the swelling profiles of the FT45 and FP45 tablets. The porosity of a tablet has a significant

effect on the swelling behaviour where tablets with a lower porosity tend to swell to a larger extent due to the slow hydration process in such tablets. The smaller rate of water uptake of the tablet with the lower porosity, compared to that of the tablet with a higher porosity, results in a smaller rate of swelling. Therefore, the tablet swells to a greater extent before the disintegration process occurs. 

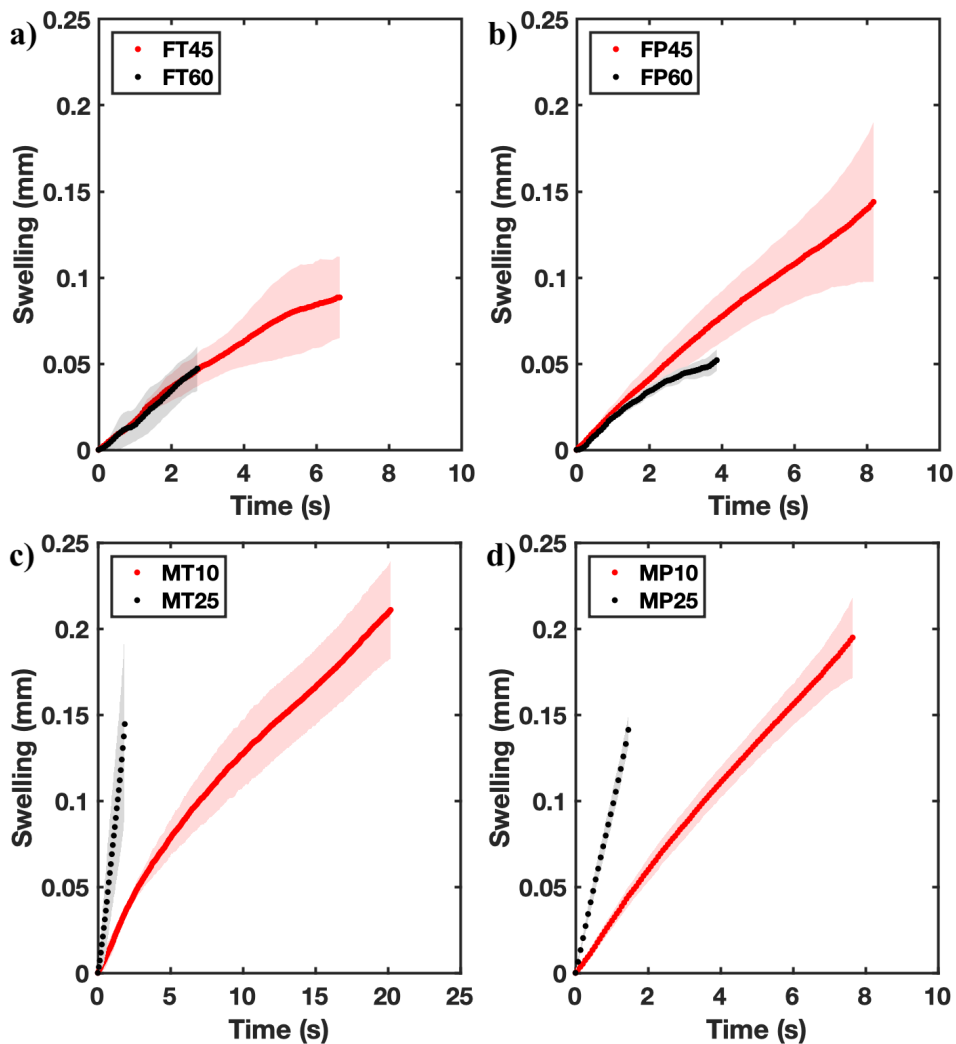

Figure 12: The average swelling profiles of all the FCC based tablets ( $\mathrm{a}$ and $\mathrm{b}$ ) and the MCC based tablets (c and d). The shaded area represents the standard deviation for each set.

\subsection{Linking Porosity to Transport Kinetics}

A power law model, which has been previously implemented for the investigation of drug release kinetics (Ritger \& Peppas, 1987; Siepmann \& Siepmann, 2013), can be used to compare liquid transport kinetics of different tablets. This simple mathematical model is a correlation of the front movement of a liquid and its transport kinetics in a porous medium, which can be defined as follows (Yassin et al., 2015a):

$$
y(t)=k t^{m}
$$

where $y$ is the displacement of liquid penetration front or liquid penetration 
depth as a function of time $t, k$ is a fitting parameter related to the tablet's geometry and structure and the exponent $m$ characterises the mechanism of mass transport process (Markl \& Zeitler, 2017). The value of $m$ of the liquid penetration, $m_{\mathrm{LP}}$, can be used to differentiate between different types of mass transport: pure Darcy flow $(m=0.5)$, case II transport $(m=1)$ or anomalous transport (an intermediate value of $m$ between 0.5 and 1) (Yassin et al., 2015a).

Fitting the power law to the experimental data of all the tablets yields an $m$ value ranging between 0.5 and 1 (Figure 13) indicating an anomalous transport in all the tablets, which is a combination of Darcy flow, driven by a capillary pressure gradient, and case II transport, driven by an activity gradient (Markl \& Zeitler, 2017). The activity gradient is the impact of swelling on the liquid transport into the tablet matrix due to hindered transport as a result of some effects including hydrogel formation, increased tortuosity, decreased porosity or enhanced swelling behaviour (Yassin et al., 2015a). 

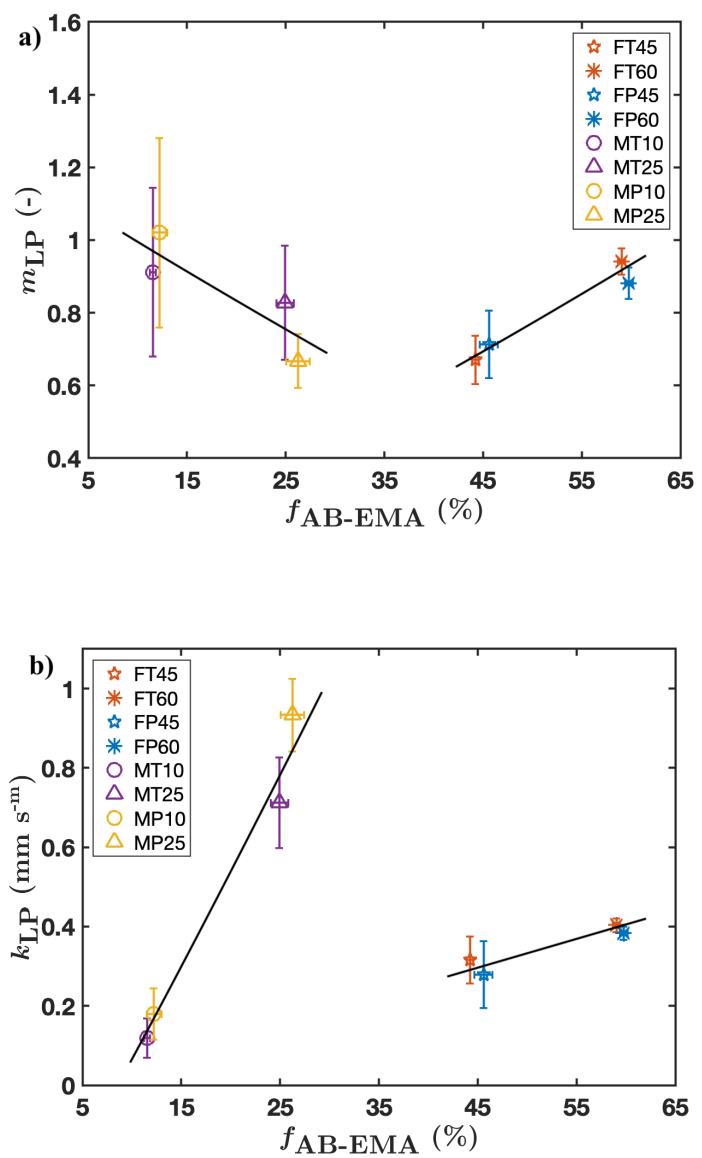

Figure 13: (a) $m_{\mathrm{LP}}$ parameter and (b) $k_{\mathrm{LP}}$ parameter of liquid penetration as a function of the THz-TDS porosity.

Unsurprisingly, the $k$ parameter of liquid penetration, $k_{\mathrm{LP}}$ parameter, is linearly related to the tablet porosity (Figure 13), where the difference of the $k_{\mathrm{LP}}$ between the low and high porosity samples is more significant for the MCC based tablets. The $k_{\mathrm{LP}}$ parameter was also investigated as a function of the pore diameter at the interparticle pore volume peaks. Figure 14 shows that the $k_{\mathrm{LP}}$ parameter increases with an increase in the pore diameter within each set of tablets with the same filler (i.e. FCC or MCC). 


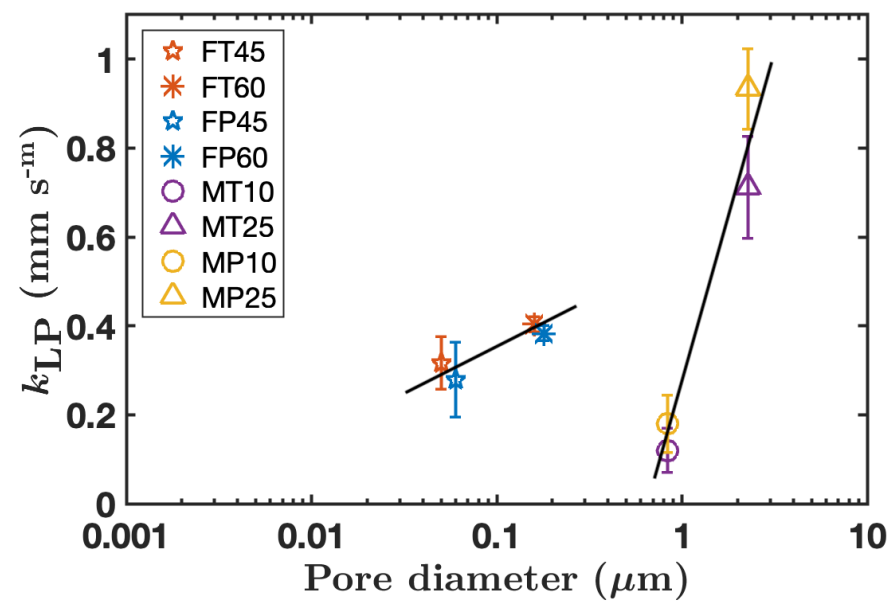

Figure 14: The $k$ parameter of liquid penetration, $k_{\mathrm{LP}}$, as a function of the pore diameter.

The power law was also used for fitting the experimental data of the swelling in order to determine the rate of swelling, $k_{\mathrm{Sw}}$. The swelling rate directly correlates with the porosity for the MCC based tablets (Figure 15). This data also indicates that the $k_{\mathrm{SW}}$ is almost constant for the FCC based tablets despite the difference in API and porosity. These results are in line with the fact that the only material with swelling capacity is the CCS in the FCC based formulations. The swelling is thus fully controlled by the amount of CCS, whereas the liquid uptake is driven by the FCC material and the interparticle pores created during compaction. The MCC based formulations are more complex as both CCS and MCC swell. The increase in the rate of swelling with increasing porosity indicates that the MCC based formulations are performance-limited by its ability to take up liquid. 


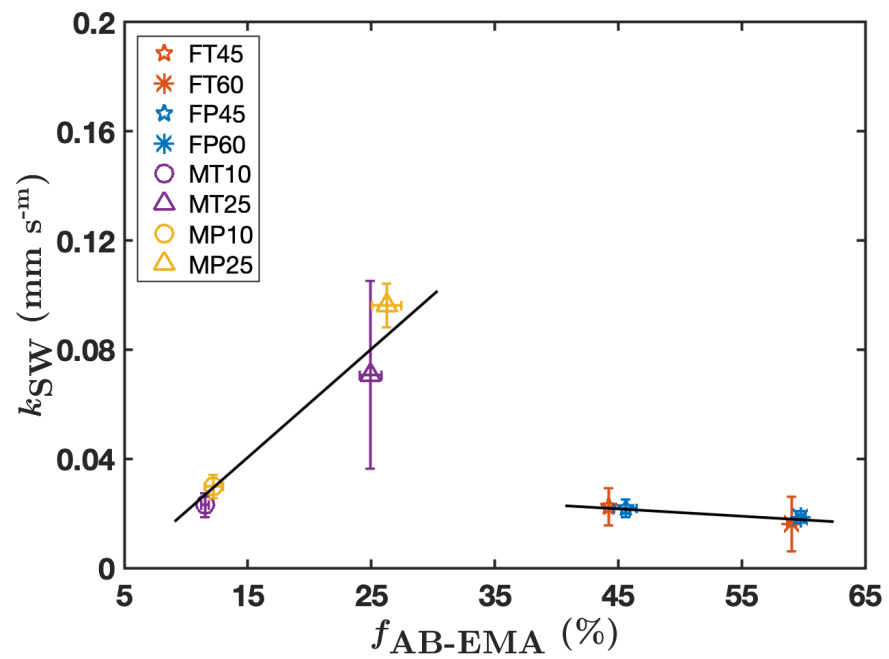

Figure 15: The $k_{\mathrm{SW}}$ parameter of swelling as a function of the $\mathrm{THz}$ porosity.

\section{Conclusions}

The terahertz techniques have been used to study the effect of the microstructure characteristics on the liquid transport and swelling kinetics in complex pharmaceutical powder compacts. THz-TDS in a transmission setting provided fast, non-destructive and contactless measurements of the structural properties of porous media, i.e. the porosity, pore shape and the alignment of pores within compacted tablets. TPI was able to capture fast liquid transport process and provided a quantitative analysis of the liquid penetration and swelling kinetics. This proves useful in monitoring the rapid liquid imbibition and swelling behaviour of FDTs. Combining the porosity measurements with the liquid transport and swelling data revealed that the disintegration performance of MCC-based tablets is liquid uptake limited, whereas the FCC based tablets are limited by its ability to swell. The swelling ability can be decoupled from the liquid uptake rate for the FCC based tablets enabling the control of each of the mechanisms independently.

This work can help formulation scientists in the pharmaceutical industry enhance the design of reproducible formulations and dosage forms. It is important 
to consider the effects of the liquid transport in the design of pharmaceutical dosage forms. This helps in a better selection of the materials incorporated in the dosage forms to achieve the desired disintegration process and hence optimise the performance of the drug in the body. For instance, the disintegration can be controlled through disintegrant concentration regardless of compaction when using a non-swelling porous filler. On the other hand, using the more widely established MCC, the liquid transport, and hence disintegration, become limited at high compaction pressure, i.e. low porosity, as swelling MCC particles will restrict further supply of dissolution medium into the tablet due to the potential blockage of the small pore space with the swelling of the MCC particles.

\section{Acknowledgements}

We would like to thank Johnson Matthey and the U.K. Engineering and Physical Sciences Research Council (EPSRC) for their funding, and Omya International AG for providing the FCC and performing the surface area and porosimetric analyses.

\section{References}

Bandari, S., Mittapalli, R. K., Gannu, R., \& Rao, Y. M. (2014). Orodispersible tablets: An overview. Asian Journal of Pharmaceutics, 2.

Bawuah, P., Ervasti, T., Tan, N., Zeitler, J. A., Ketolainen, J., \& Peiponen, K.-E. (2016). Noninvasive porosity measurement of biconvex tablets using terahertz pulses. International Journal of Pharmaceutics, 509, 439-443.

Bawuah, P., Karttunen, A.-P., Markl, D., Ridgway, C., Korhonen, O., Gane, P., Zeitler, J. A., Ketolainen, J., \& Peiponen, K.-E. (2018). Investigating elastic relaxation effects on the optical properties of functionalised calcium carbonate compacts using optics-based Heckel analysis. International Journal of Pharmaceutics, 544, 278-284. 
Bawuah, P., Mendia, A. P., Silfsten, P., Pääkkönen, P., Ervasti, T., Ketolainen, J., Zeitler, J. A., \& Peiponen, K.-E. (2014a). Detection of porosity of pharmaceutical compacts by terahertz radiation transmission and light reflection measurement techniques. International Journal of Pharmaceutics, 465, 7076.

Bawuah, P., Silfsten, T., P.and Ervasti, Ketolainen, J., Zeitler, J. A., \& Peiponen, K.-E. (2014b). Non-contact weight measurement of flat-faced pharmaceutical tablets using terahertz transmission pulse delay measurements. International Journal of Pharmaceutics, 476, 16-22.

Chakraborty, M., Ridgway, C., Bawuah, P., Markl, D., Gane, P. A. C., Ketolainen, J., Zeitler, J. A., \& Peiponen, K.-E. (2017). Optics-based compressibility parameter for pharmaceutical tablets obtained with the aid of the terahertz refractive index. International Journal of Pharmaceutics, 525, $85-91$.

Chandrasekhar, R., Hassan, Z., AlHusban, F., Smith, A. M., \& Mohammed, A. R. (2009). The role of formulation excipients in the development of lyophilised fast-disintegrating tablets. European Journal of Pharmaceutics and Biopharmaceutics, 72, 119-129.

Chang, S.-Y., \& Sun, C. C. (2017). Superior Plasticity and Tabletability of Theophylline Monohydrate. Mol. Pharmaceutics, 14, 2047-2055.

Chauhan, K., Solanki, R., \& Sharma, S. (2018). A review on fast dissolving tablet. International Journal of Applied Pharmaceutics, 10, 1.

Corveleyn, S., \& Remon, J. P. (1997). Formulation and production of rapidly disintegrating tablets by lyophilisation using hydrochlorothiazide as a model drug. International Journal of Pharmaceutics, 152, 215-225.

Desai, C. V., P. M.and Liew, \& Heng, P. W. S. (2012). Understanding disintegrant action by visualization. Journal of Pharmaceutical Sciences, 101, $2155-2164$. 
Desai, P. M., Liew, C. V., \& Heng, P. W. S. (2016). Review of disintegrants and the disintegration Phenomena. Journal of Pharmaceutical Sciences, 105, 2545-2555.

Djemai, A., \& Sinka, I. C. (2006). NMR imaging of density distributions in tablets. International Journal of Pharmaceutics, 319, 55-62.

Dukić-Ott, A., Remon, J. P., Foreman, P., \& Vervaet, C. (2007). Immediate release of poorly soluble drugs from starch-based pellets prepared via extrusion/spheronisation. European Journal of Pharmaceutics and Biopharmaceutics, 67, 715-724.

Ekmekciyan, N., Tuglu, T., El-Saleh, F., Muehlenfeld, C., Stoyanov, E., \& Quodbach, J. (2018). Competing for water: A new approach to understand disintegrant performance. International Journal of Pharmaceutics, 548, 491499 .

European Medicines Agency (2008). ICH topic Q4B annex 5: Disintegration test general chapter. London: UK.

Fu, Y., Yang, S., Jeong, S. H., Kimura, S., \& Park, K. (2004). Orally Fast Disintegrating Tablets: Developments, Technologies, Taste-Masking and Clinical Studies. Critical Reviews in Therapeutic Drug Carrier Systems, 21, 433-476.

Gaikwad, V. L., Sharma, S. N., Bhatia, M. S., \& Mahadik, K. R. (2018). Development of controlled release cellulose-pellets based multicore tablets. European Polymer Journal, 105, 389-397.

Gane, P. A., Kettle, J. P., Matthews, G. P., \& Ridgway, C. J. (1996). Void space structure of compressible polymer spheres and consolidated calcium carbonate paper-coating formulations. Industrial and Engineering Chemistry Research, 35, 1753-1764.

Gulsun, T., Cayli, Y. A., Izat, N., Cetin, M., Oner, L., \& Sahin, S. (2018). Development and evaluation of terbutaline sulfate orally disintegrating tablets 
by direct compression and freeze drying methods. Journal of Drug Delivery Science and Technology, 46, 251-258.

Han, R., Yang, Y., Li, X., \& Ouyang, D. (2018). Predicting oral disintegrating tablet formulations by neural network techniques. Asian Journal of Pharmaceutical Sciences, 13, 336-342.

Kaerger, J. S., Edge, S., \& Price, R. (2004). Influence of particle size and shape on flowability and compactibility of binary mixtures of paracetamol and microcrystalline cellulose. European Journal of Pharmaceutical Sciences, 22, 173-179.

Markl, D., Bawuah, P., Ridgway, C., van den Ban, S., Goodwin, D. J., Ketolainen, J., Gane, P., Peiponen, K.-E., \& Zeitler, J. A. (2018a). Fast and nondestructive pore structure analysis using terahertz time-domain spectroscopy. International Journal of Pharmaceutics, 537, 102-110.

Markl, D., Sauerwein, J., Goodwin, D. J., van den Ban, S., \& Zeitler, J. A. (2017a). Non-destructive determination of disintegration time and dissolution in immediate release tablets by terahertz transmission measurements. Pharmaceutical Research, 34, 1012-1022.

Markl, D., Strobel, A., Schlossnikl, R., Bøtker, J., Bawuah, P., Ridgway, C., Rantanen, J., Rades, T., Gane, P., Peiponen, K.-E., \& Zeitler, J. A. (2018b). Characterisation of pore structures of pharmaceutical tablets: A review. International Journal of Pharmaceutics, 538, 188-214.

Markl, D., Wang, P., Ridgway, C., Karttunen, A.-P., Bawuah, P., Ketolainen, J., Gane, P., Peiponen, K.-E., \& Zeitler, J. A. (2018c). Resolving the rapid water absorption of porous functionalised calcium carbonate powder compacts by terahertz pulsed imaging. Chemical Engineering Research and Design, 132, 1082-1090.

Markl, D., Wang, P., Ridgway, C., Karttunen, A.-P., Chakraborty, M., Bawuah, P., Pääkkönen, P., Gane, P., Ketolainen, J., Peiponen, K.-E., \& Zeitler, J. A. 
(2017b). Characterization of the pore Structure of functionalized calcium carbonate tablets by terahertz time-domain spectroscopy and X-ray computed microtomography. Journal of Pharmaceutical Sciences, 106, 1586-1595.

Markl, D., Yassin, S., Wilson, D. I., Goodwin, D. J., Anderson, A., \& Zeitler, J. A. (2017c). Mathematical modelling of liquid transport in swelling pharmaceutical immediate release tablets. International Journal of Pharmaceutics, 526, 1-10.

Markl, D., \& Zeitler, J. A. (2017). A review of disintegration mechanisms and measurement techniques. Pharmaceutical Research, 34, 890-917.

Mesnier, X., Althaus, T. O., Forny, L., Niederreiter, G., Palzer, S., Hounslow, M. J., \& Salman, A. D. (2013). A novel method to quantify tablet disintegration. Powder Technology, 238, 27-34.

Nishiyama, T., Ogata, T., \& Ozeki, T. (2016). Preparation of bitter tastemasking granules of lafutidine for orally disintegrating tablets using waterinsoluble/soluble polymer combinations. Journal of Drug Delivery Science and Technology, 32, 38-42.

Nogami, H., Hasegawa, J., \& Miyamoto, M. (1967). Studies of powdered preperations XX disintegration of the aspirin tablets containing starches as disintegrating agent. Chem Pharm Bull, 15, 279-289.

Nogami, H., Nagai, T., \& Uchida, H. (1966). Studies on powdered preparations. XIV. Wetting of powder bed and disintegration time of tablet. Chemical and Pharmaceutical Bulletin, 14, 152-158.

Obradovic, J., Collins, J. H. P., Hirsch, O., Mantle, M. D., Johns, M. L., \& Gladden, L. F. (2007). The use of THz time-domain reflection measurements to investigate solvent diffusion in polymers. Polymer, 48, 3494-3503.

Pabari, R. M., \& Ramtoola, Z. (2012). Application of face centred central composite design to optimise compression force and tablet diameter for the for- 
mulation of mechanically strong and fast disintegrating orodispersible tablets. International Journal of Pharmaceutics, 430, 18-25.

Parkash, V., Maan, S., Deepika, Yadav, S. K., Hemlata, \& Jogpal, V. (2011). Fast disintegrating tablets: Opportunity in drug delivery system. Journal of Advanced Pharmaceutical Technology \&3 Research, 2, 223.

Paul, S., \& Sun, C. C. (2017). Gaining insight into tablet capping tendency from compaction simulation. Int. J. Pharm., 524, 111-120.

Peppas, N., \& Colombo, P. (1989). Development of disintegration forces during water penetration in porous pharmaceutical systems. Journal of Controlled Release, 10, 245-250.

Pickwell, E., \& Wallace, V. P. (2006). Biomedical applications of terahertz technology. Journal of Physics D: Applied Physics, 39, R301-R310.

Quodbach, J., \& Kleinebudde, P. (2014). Systematic classification of tablet disintegrants by water uptake and force development kinetics. Journal of Pharmacy and Pharmacology, 66, 1429-1438.

Quodbach, J., \& Kleinebudde, P. (2016). A critical review on tablet disintegration. Pharmaceutical Development and Technology, 47, 1-12.

Quodbach, J., Moussavi, A., Tammer, R., Frahm, J., \& Kleinebudde, P. (2014). Tablet disintegration studied by high-resolution real-time magnetic resonance imaging. Journal of Pharmaceutical Sciences, 103, 249-255.

Ridgway, C., Bawuah, P., Markl, D., Zeitler, J. A., Ketolainen, J., Peiponen, K.-E., \& Gane, P. (2017). On the role of API in determining porosity, pore structure and bulk modulus of the skeletal material in pharmaceutical tablets formed with MCC as sole excipient. International Journal of Pharmaceutics, $526,321-331$.

Ridgway, C. J., Gane, P. A. C., \& Schoelkopf, J. (2004). Modified calcium carbonate coatings with rapid absorption and extensive liquid uptake capac- 
ity. Colloids and Surfaces A: Physicochemical and Engineering Aspects, 236, $91-102$.

Ritger, P. L., \& Peppas, N. A. (1987). A simple equation for description of solute release I. Fickian and non-fickian release from non-swellable devices in the form of slabs, spheres, cylinders or discs. Journal of Controlled Release, $5,23-36$.

Siepmann, J., \& Siepmann, F. (2013). Mathematical modeling of drug dissolution. International Journal of Pharmaceutics, 453, 12-24.

Stirnimann, T., Di Maiuta, N., Gerard, D. E., Alles, R., Huwyler, J., \& Puchkov, M. (2013). Functionalized calcium carbonate as a novel pharmaceutical excipient for the preparation of orally dispersible tablets. Pharmaceutical Research, 30, 1915-1925.

Thoorens, G., Krier, F., Leclercq, B., Carlin, B., \& Evrard, B. (2014). Microcrystalline cellulose, a direct compression binder in a quality by design environment-A review. International Journal of Pharmaceutics, 473, 6472 .

Tritt-Goc, J., \& Kowalczuk, J. (2002). In situ, real time observation of the disintegration of paracetamol tablets in aqueous solution by magnetic resonance imaging. European Journal of Pharmaceutical Sciences, 15, 341-346.

Yalkowsky, S. H., He, Y., \& Jain, P. (2010). In Handbook of Aqueous Solubility Data (pp. 395, 492). Florida: US: CRC Press.

Yassin, S., Goodwin, D. J., Anderson, A., Sibik, J., Wilson, D. I., Gladden, L. F., \& Zeitler, J. A. (2015a). The Disintegration process in microcrystalline cellulose based tablets, part 1: Influence of temperature, porosity and superdisintegrants. Journal of Pharmaceutical Sciences, 104, 3440-3450.

Yassin, S., Su, K., Lin, H., Gladden, L. F., \& Zeitler, J. A. (2015b). Diffusion and swelling measurements in pharmaceutical powder compacts using terahertz pulsed imaging. Journal of Pharmaceutical Sciences, 104, 1658-1667. 
679

Zeitler, J. A. (2016). Pharmaceutical Terahertz Spectroscopy and Imaging. In A. Muellertz, T. Rades, \& Y. Perrie (Eds.), Analytical Techniques in the Pharmaceutical Sciences (pp. 171-222). New York, NY: Springer: Advances in Delivery Science and Technology.

Zeitler, J. A., \& Shen, Y.-C. (2012). Industrial Applications of Terahertz Imaging. In Terahertz Spectroscopy and Imaging (pp. 451-489). Berlin, Heidelberg: Springer. 
Appendix A. Supporting Information

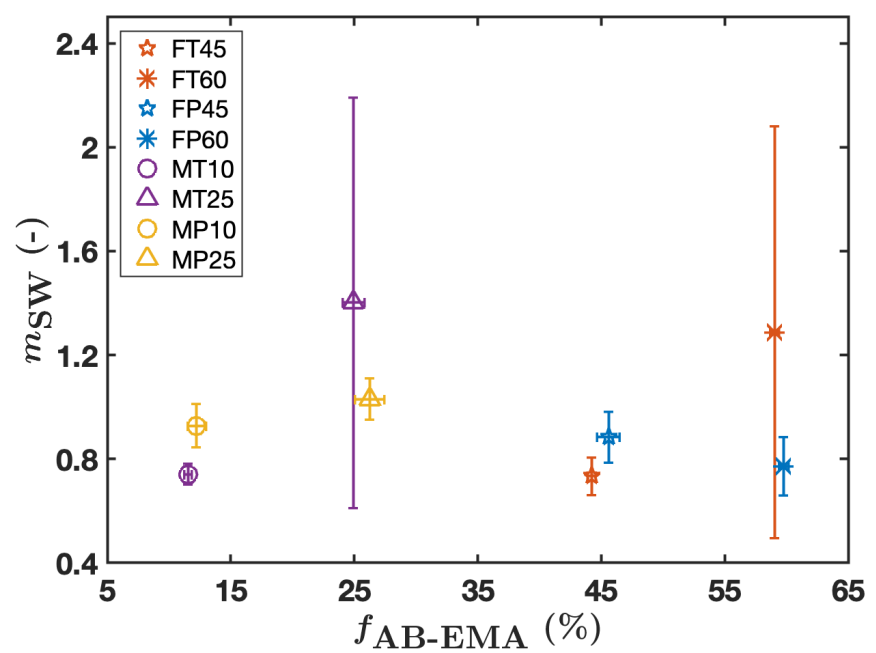

Figure A.16: The $m_{\mathrm{SW}}$ parameter of swelling as a function of the $\mathrm{THz}$ porosity. 

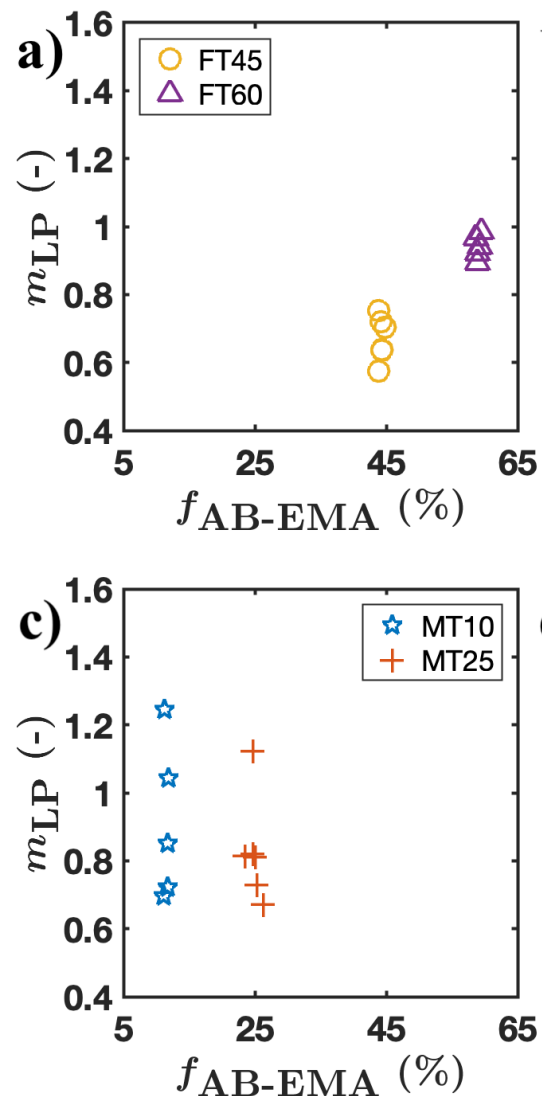
Porosity
Appendix A.1. Kinetics Parameters of Individual Tablets as a Function of THz
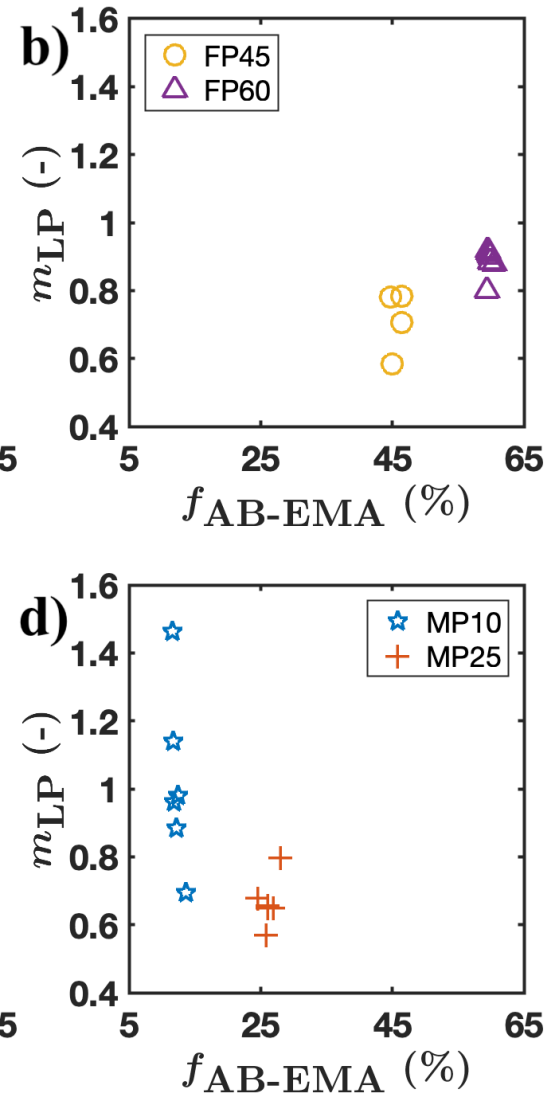

Figure A.17: The $m_{\mathrm{LP}}$ parameter of liquid penetration as a function of the $\mathrm{THz}$ porosity for the FCC based tablets ( $a$ and b) and the MCC based tablets (c and d). 

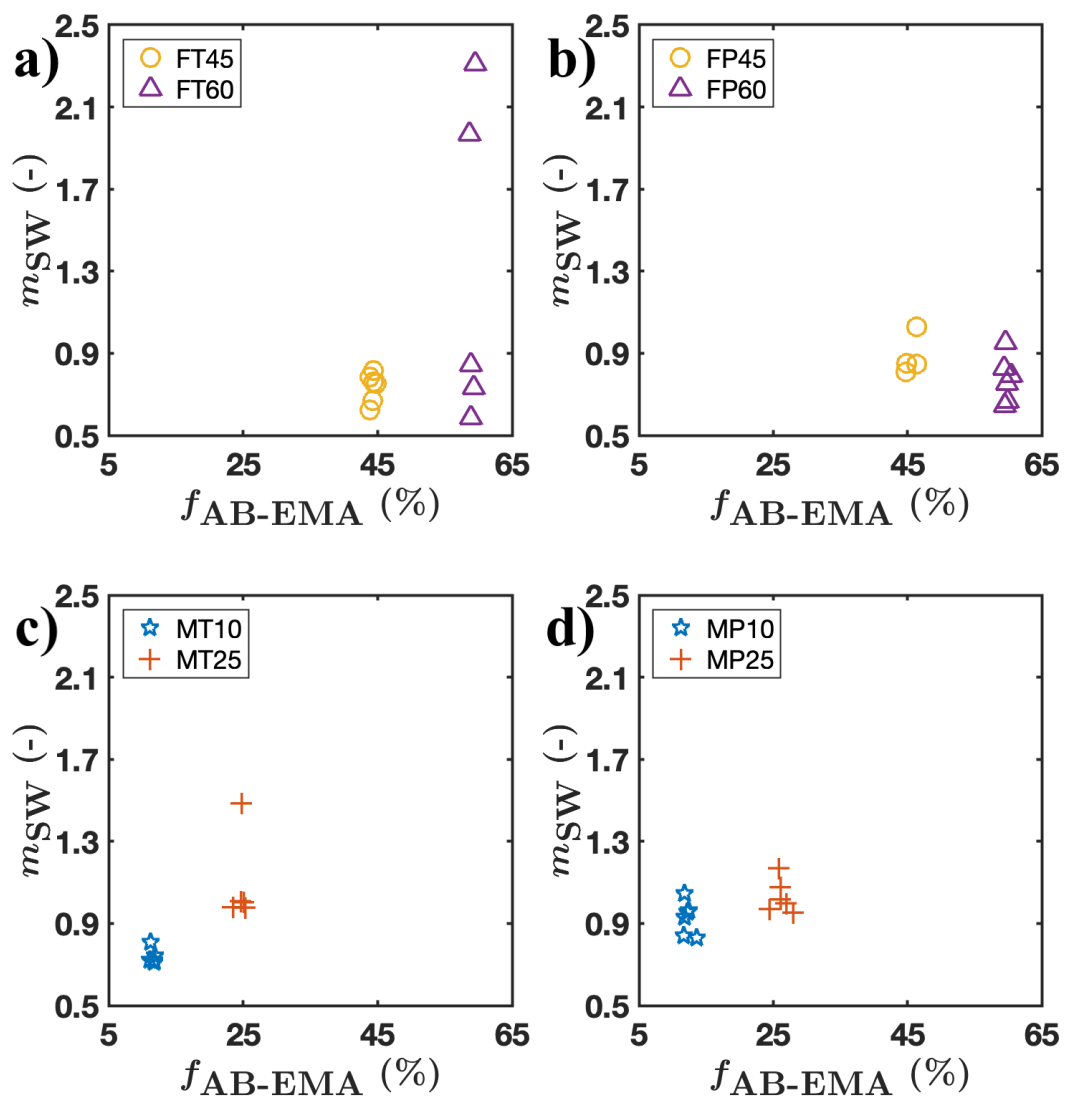

Figure A.18: The $m_{\mathrm{SW}}$ parameter of swelling as a function of the THz porosity for the FCC based tablets ( $\mathrm{a}$ and b) and the MCC based tablets (c and d). 

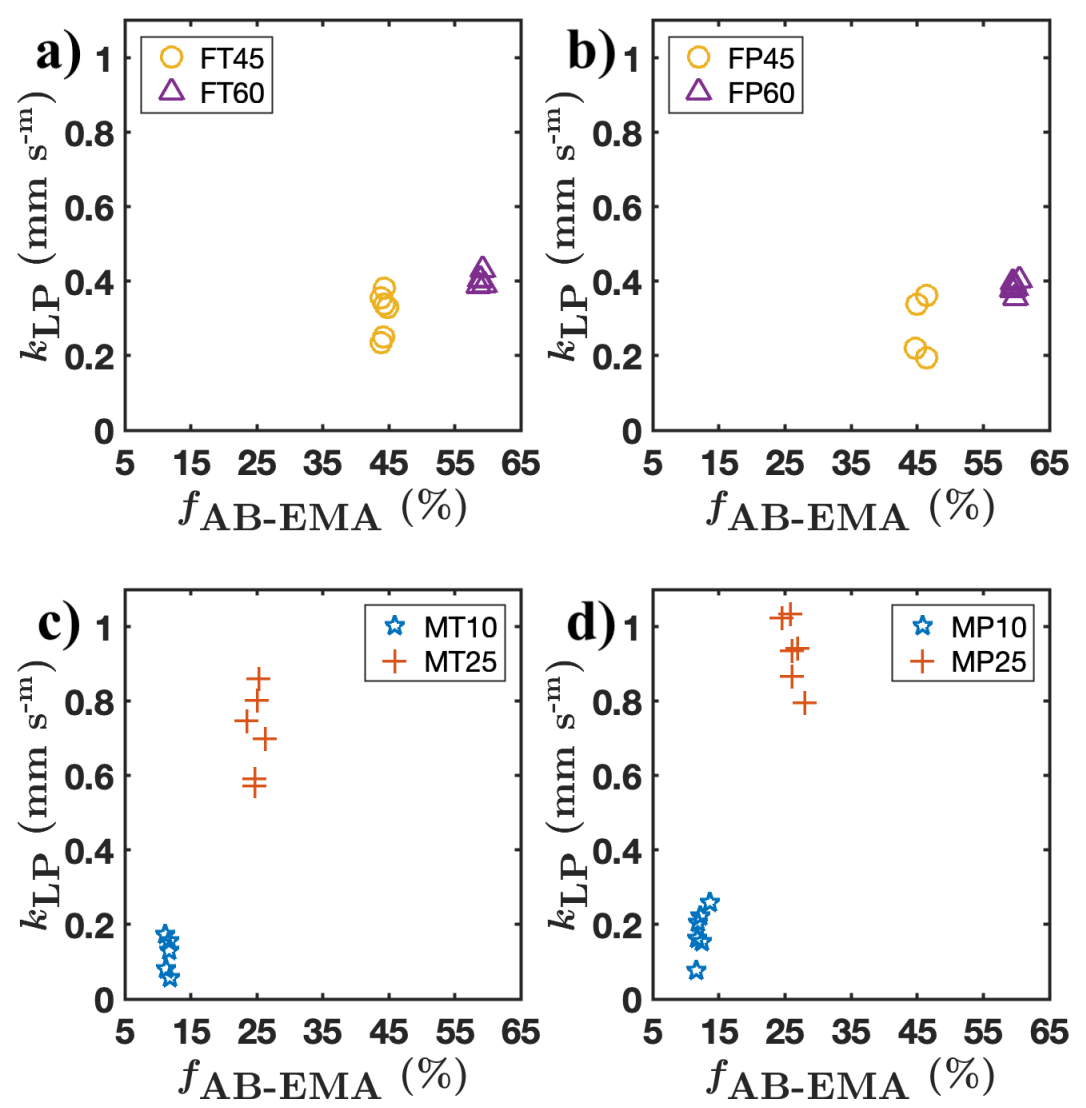

Figure A.19: The $k_{\mathrm{LP}}$ parameter of liquid penetration as a function of the THz-TDS porosity for the FCC based tablets ( $\mathrm{a}$ and b) and the MCC based tablets (c and d). 

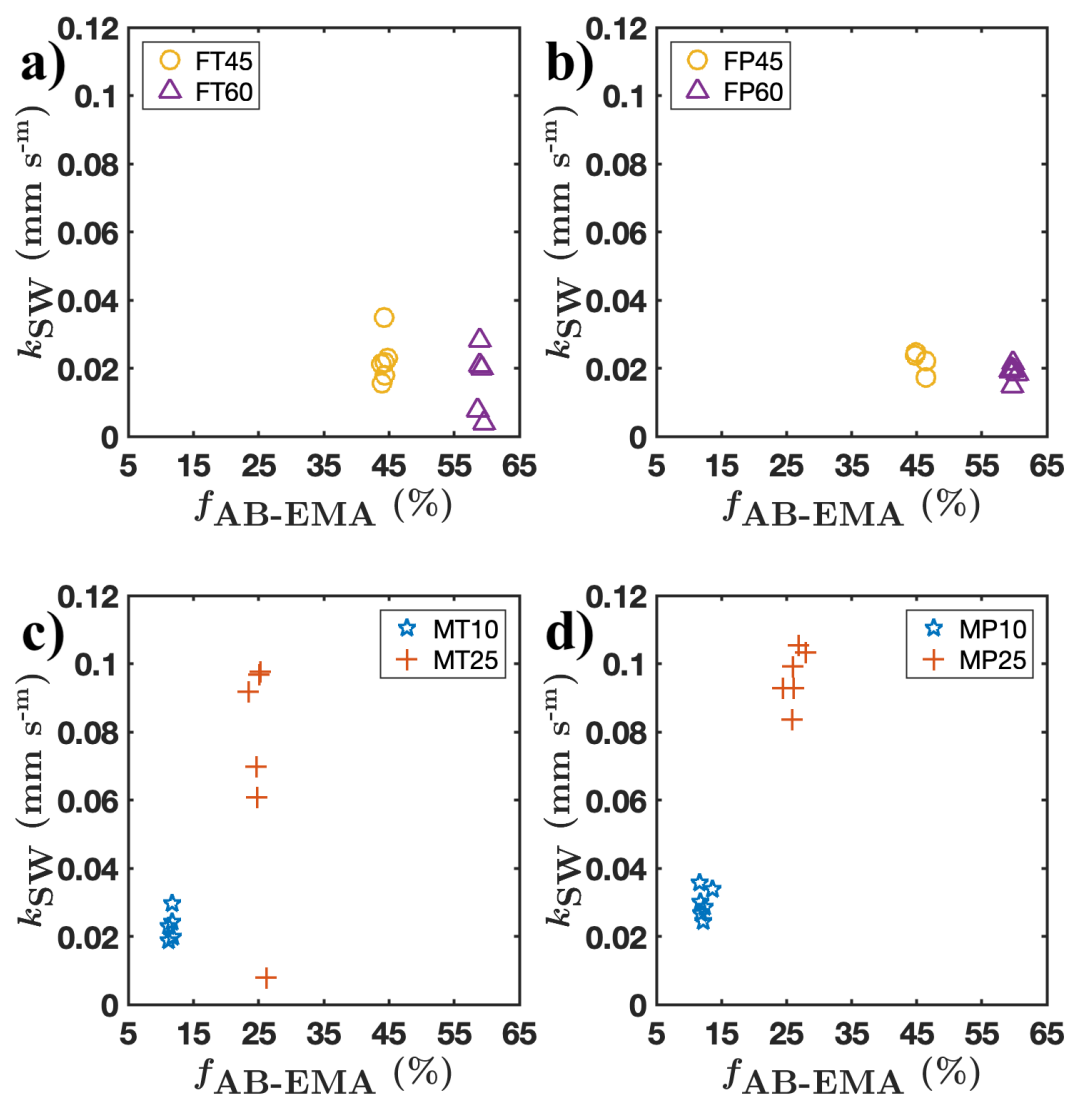

Figure A.20: The $k_{\mathrm{SW}}$ parameter of swelling as a function of the THz porosity for the FCC based tablets ( $\mathrm{a}$ and $\mathrm{b}$ ) and the MCC based tablets (c and d). 


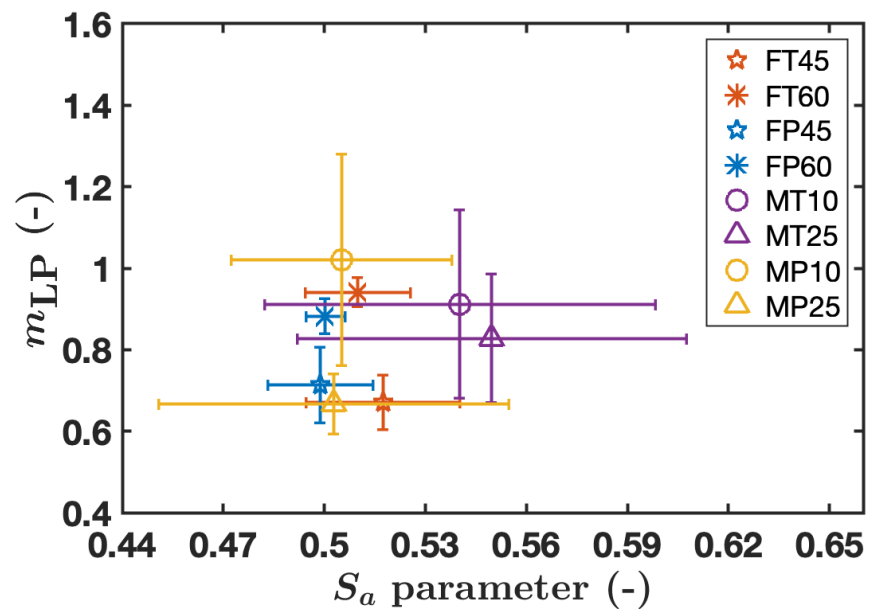

Figure A.21: The $m_{\mathrm{LP}}$ parameter of liquid penetration as a function of $S_{\mathrm{a}}$ parameter.

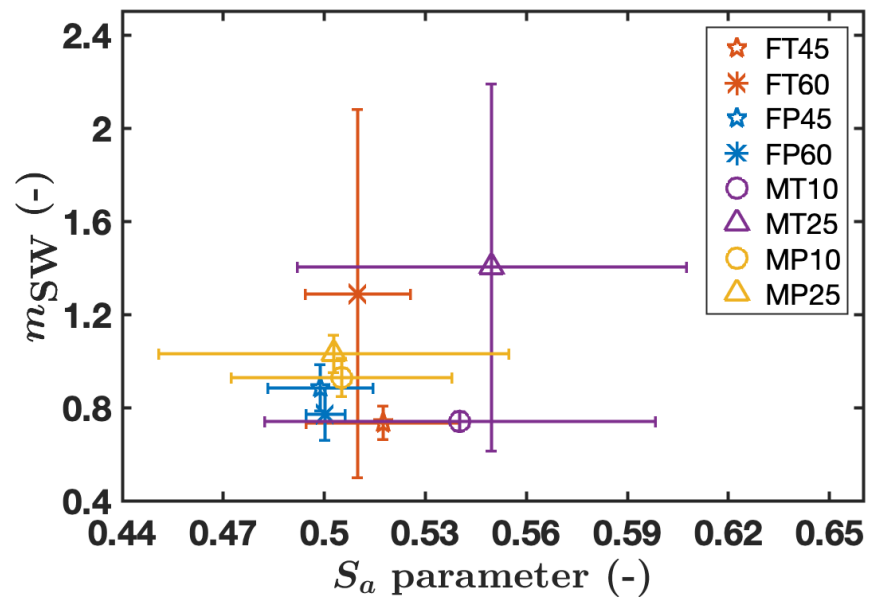

Figure A.22: The $m_{\mathrm{SW}}$ parameter of swelling as a function of $S_{\mathrm{a}}$ parameter. 


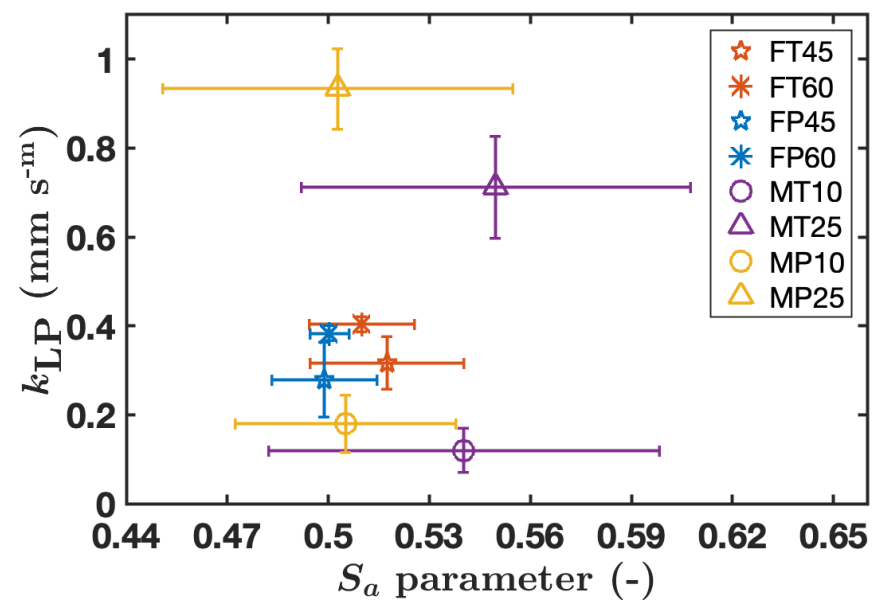

Figure A.23: The $k_{\mathrm{LP}}$ parameter of liquid penetration as a function of $S_{\mathrm{a}}$ parameter.

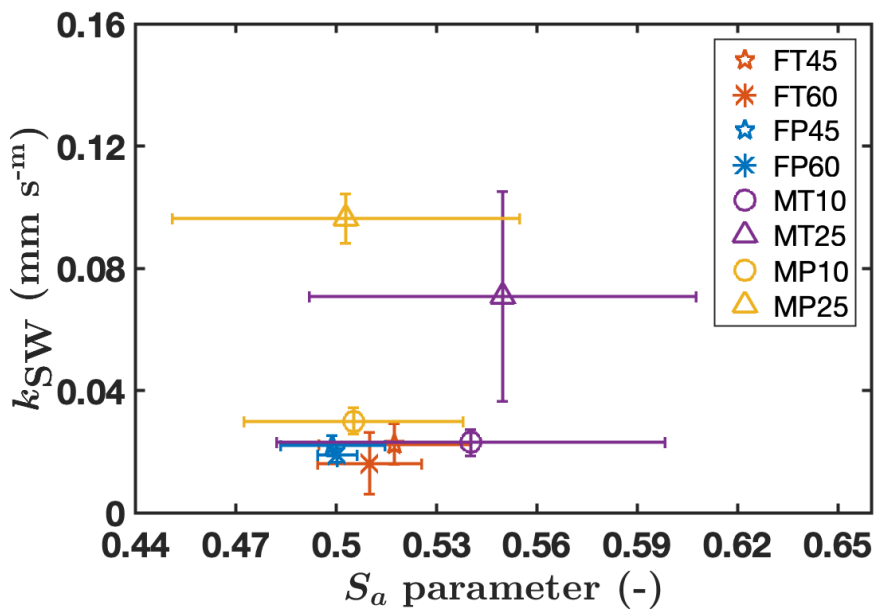

Figure A.24: The $k_{\mathrm{SW}}$ parameter of swelling as a function of $S_{\mathrm{a}}$ parameter.

Appendix A.3. Kinetics Parameters as a Function of Pore Diameter

The kinetics parameters were plotted against the pore size at the interparticle pore volume peaks for the FCC and MCC based tablets. 


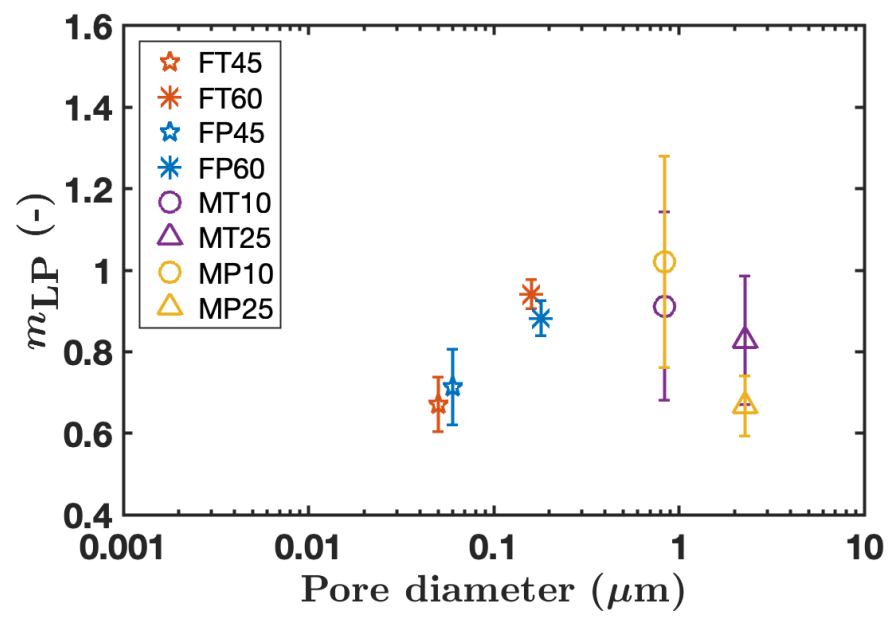

Figure A.25: The $m_{\mathrm{LP}}$ parameter of liquid penetration as a function of the pore diameter.

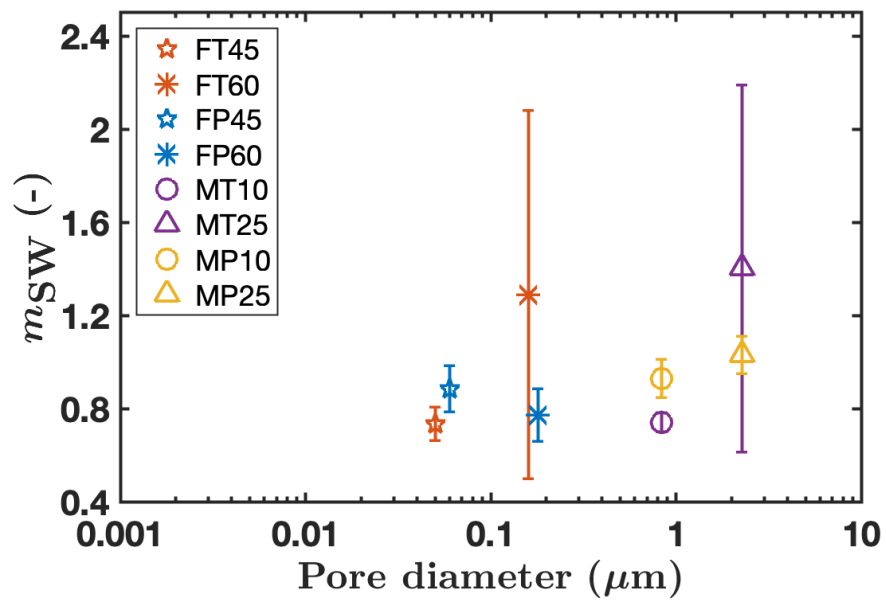

Figure A.26: The $m_{\mathrm{SW}}$ parameter of swelling as a function of the pore diameter. 


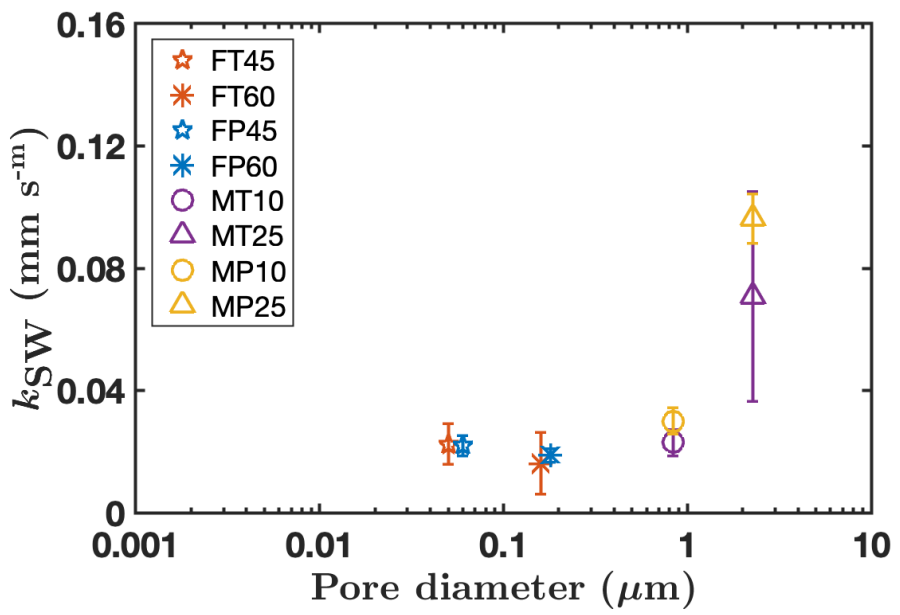

Figure A.27: The $k_{\mathrm{SW}}$ parameter of swelling as a function of the pore diameter. 